\title{
The Effects of Cohesive Strength And Toughness on Mixed-Mode Delamination of BeAM-Like GeOMETRIES
}

\author{
J. P. Parmigiani ${ }^{1 *}$ and M. D. Thouless ${ }^{1,2}$ \\ ${ }^{1}$ Department of Mechanical Engineering \\ ${ }^{2}$ Department of Materials Science \& Engineering \\ University of Michigan \\ Ann Arbor, MI 48109
}

January 30, 2007

\begin{abstract}
Cohesive-zone models have been used to study the effects of strength and toughness on the delamination of beam-like geometries. The conditions under which linear-elastic interfacial mechanics provide a good frame-work for predicting failure of such systems have been studied. It has been determined that the phase angle derived from LEFM calculations provides an excellent description of the partitioning between the mode-I and mode-II energy-release rates over a wide range of fracture-length scales. In particular, the nominal phase angle can be a useful parameter, even when the fracture-length scale is so large that the interface stresses do not exhibit the expected inverse-squareroot dependence. The analysis has also shown that nominal phase angles with a magnitude greater than $90^{\circ}$ can have physical significance, provided the interface layer is thick enough to accommodate compression without crack-surface contact. Finally, the role of modulus mismatch has been studied. A length scale introduced by the cohesive strength allows a crack-tip phase angle to be established, when LEFM predicts oscillating stress fields at the crack tip. This crack-tip phase angle is shifted from the nominal phase angle based on a characteristic geometrical length by an amount that depends on the cohesive parameters of the interface and the modulus mismatch. It has been shown that, as a result of this shift, both modulus mismatch parameters can influence the strength of an interface.
\end{abstract}

${ }^{*}$ Current address: Department of Mechanical Engineering, Oregon State University, Corvallis, OR 97331 


\section{Introduction}

\subsection{General background}

Historically, two distinct approaches for analyzing the fracture of interfaces and delamination of layered materials have been developed. One, based on the Inglis approach to fracture [1], uses a strength-based failure criterion. The stress (or traction) distribution along an interface is computed, and delamination is assumed to occur when the stress at the crack tip reaches a critical value of the cohesive strength (e.g., [2]). The other, based on the Griffith approach to fracture [3], has become universally adopted during the last two decades, and is the basis of interfacial fracture mechanics with its assumptions of linear elasticity and pre-existing flaws. The energy-release rate (or crack-driving force) is computed for the body. This quantity can be considered to be the energy density at the crack tip, and delamination is assumed to occur when this quantity reaches a critical value of the toughness (e.g., [4]).

The bonding across an interface in an engineering, physical or biological system can be regarded as a set of tractions that varies as a function of the separation between the two adherends, and that can be characterized by an appropriate tractionseparation law. For fracture to occur, the local stress across the interface must exceed the cohesive strength of the interface (the peak stress in the traction-separation law), and the energy density supplied to the crack tip must be greater than the toughness of the interface (the area under the traction-separation law). In other words, the failure criterion for the strength-based approach and the failure criterion for the toughnessbased approach must be met simultaneously. While strength-based approaches tend not to include considerations of energy-release rate, energy-based approaches of fracture mechanics do have an implicit assumption that the strength criterion is always satisfied, because the stress field at a crack tip in an elastic material is singular.

The tractions across an interface may be formed by direct chemical bonding between the adherends; the traction-separation law then represents the atomic bonding. The tractions may also be formed by physical bonding arising from van der Waals forces, electrostatic or magnetic attraction. Bonding at an interface may also be caused by an interphase. This interphase could consist of individual bonding molecules, such as proteins or other organic chains, or it could consist of a bulk second phase chemically bonded to the adherends, such as a metal or an adhesive layer. Depending on the nature of the interphase, the traction-separation law would either be associated with uncoiling the molecules and breaking any ligand-receptor bonds [5], or with the deformation of the bulk layer up to the point of fracture. Obviously, in the latter case, the characteristics of such a traction-separation law might

be expected to change with geometrical parameters $[6,7]$. Traditional approaches of interfacial fracture mechanics also have to accommodate the issue of a geometry- 
dependent toughness when used for the analysis of adhesive joints. The toughness can depend on the thickness of the adhesive layer, so that the use of fracture mechanics for design purposes is limited to adhesive layers of comparable thicknesses to those for which the toughness was assessed. Another form of interphase that can be considered for interfaces is a bulk second phase physically bonded to the adherends. For example, stiction in MEMS devices may be caused by capillary condensation at the interface between an actuator and a substrate [8]. The capillary pressure provides tractions across the interface, and crack advance along the interface involves fluid flow within the condensate. Lubricated interfaces can also be regarded as fracturing in a similar fashion. Model experiments have shown that when the liquid phase is very thin, crack advance is dependent on the difference in surface energy between the wetted interface and the unwetted interface $[9,10]$.

A particularly coherent body of literature, based on an implicit assumption of singular stresses at the crack tip, has been developed for the interfacial fracture mechanics of layered materials [11]. However, there are many practical applications in which the stresses across the interface are sufficiently limited in magnitude to justify concerns as to whether analyses based on linear elasticity are appropriate. Therefore, the goal of this paper is to explore the range of interfacial properties for which interfacial fracture mechanics can be expected to provide a good approach for engineering design and prediction, and how deviations from this range might affect the fracture behavior. In the next section of this paper, interfacial fracture mechanics is reviewed, with a particular focus on the implicit assumptions and approaches used in design. In the following section, the rationale behind using cohesive-zone models to examine this problem is discussed, along with some details of the associated numerical modeling. In the subsequent sections, various aspects of interfacial fracture are examined, with particular foci on the role of finite cohesive strengths, the interpretation of mixed-mode fracture, delamination in the presence of compressive stresses across the interface, and the effects of modulus mismatch across the interface. All the analyses presented in this paper are for geometries in which the adherends on either side of the interface remain elastic. While the issue of plasticity in the adherends has been addressed elsewhere $[12,13]$, it is not considered here.

\subsection{Issues related to the use of interfacial fracture mechanics}

In quasi-static interfacial fracture mechanics (ignoring time-dependent aspects of crack propagation), a pre-existing crack is assumed to propagate when the energyrelease rate for the crack, $\mathcal{G}$, exceeds the appropriate toughness of the interface, $\Gamma$. In a linear-elastic system, the energy-release rate is calculated in terms of the loads, elastic constants and geometry, from a general consideration of the changes in the elastic energy and work done as the crack advances. Fracture mechanics is gener- 
ally thought of as requiring the presence of sharp cracks with singular stress fields at their tips. However, linear-elastic layered systems loaded by moments and axial loads (Fig. 1a) exhibit the useful characteristic of having steady-state solutions for the energy-release rate [14], valid when the ligament ahead of the crack tip is significantly longer than the thickness of the thinnest layer. These solutions are not predicated on any assumptions about the nature of the stress field at the crack tip. Therefore, the concept of an energy-release rate for crack propagation is rigorously correct for these geometries, even in the absence of a sharp crack tip and for any type of interface bonding. ${ }^{1}$ This has a practical effect of adding immensely to the utility of interfacial fracture mechanics, and some of this generality will be manifested in the results presented in this paper. However, there is one caveat about these steady-state solutions. Often interface cracks in layered materials are loaded normal to the interface (Fig. 1b). This results in a transverse shear acting at the crack tip, in addition to a moment. The energy-release rate associated with this transverse shear component of the load is dependent on the details of the stress field at the crack tip, as implied by the fact that a steady-state energy balance can't be used to obtain the energy-release rate associated with shear [15]. This complication results in a loss of the rigorous generality that is appropriate with only bending moments or axial loads acting at the crack tip, and means that there are important geometries for which the energy-release rate may depend on the nature of the stress field at the crack tip.

Generally, the toughness of an interface cannot be determined by calculations; it needs to be determined experimentally for the particular system of interest. Within the context of linear-elastic fracture mechanics (LEFM), this is generally done by calculating the nominal energy-release rate for crack propagation based on a macroscopic geometry (without reference to geometrical features at the crack-tip scale), elastic properties, the residual stresses, and the applied loads at which the crack propagates. This critical energy-release rate is then equated to a nominal toughness of the interface. Although, in an elastic system, the energy-release rate is constant down to the scale at which any energy dissipation may occur, the term "nominal" is used to emphasize that there may be a difference between the "actual" energy-release rate at the crack-tip scale and the energy-release rate computed at the macroscopic scale. Indeed, the fact that the nominal toughness of an interface may be different from the toughness at the crack-tip scale is a well-established axiom in LEFM, and serves as the basis for concepts of toughening following the initial insight of Irwin [16]. For example, there may be plasticity in the interphase [17], frictional effects associated with contact of the crack surfaces [18], or a lack of alignment between the crack tip and the macroscopic plane of the interface [19]. All these effects can be used as the basis of micromechanics models to relate the nominal toughness to the local toughness at the crack tip. Exploring these relationships is a valid mode of scientific

\footnotetext{
${ }^{1}$ The nature of the interfacial bonding affects the energy-release rate only if the ligament length is sufficiently short that the boundaries interact with the crack-tip stress field.
} 
inquiry, and it is certainly of interest to be able to provide a physical basis to explain phenomena such as how the nominal toughness of an adhesive interface may depend on bond-line thickness. However, with the exception of friction, these issues are not addressed in this paper. A discrepancy between "nominal" and "actual" values for the toughness of an interface can also arise because of departures from strict conditions of LEFM. Again, this is well-recognized, with some acceptable level of discrepancy forming the basis for determining whether LEFM is an appropriate tool for characterizing fracture. This is one of the issues addressed in this paper.

In a homogeneous material, the crack grows along a path in which no shear stresses act at the crack tip. However, a crack growing along an interface is generally subjected to both normal and shear modes of deformation, depending on the geometry and the nature of the loads. Therefore, fracture along an interface introduces an additional complication to the mechanics in the form of a phase angle, $\psi$, that measures the relative amounts of shear and normal deformation at the crack tip. This is of importance because there is considerable experimental evidence indicating that the fracture of an interface depends on the phase angle [20, 21, 22].

In a plane geometry containing an interface between two different isotropic materials, with moduli $E_{1}$ and $E_{2}$, and with Poisson's ratios $\nu_{1}$ and $\nu_{2}$, the mechanics depends on two non-dimensional groups of these elastic properties. These two Dundurs parameters are given by [23]

$$
\alpha=\frac{\bar{E}_{1}-\bar{E}_{2}}{\bar{E}_{1}+\bar{E}_{2}}
$$

and

$$
\beta=\frac{\bar{E}_{1} f\left(\nu_{2}\right)-\bar{E}_{2} f\left(\nu_{1}\right)}{\bar{E}_{1}+\bar{E}_{2}},
$$

where $\bar{E}=E /\left(1-\nu^{2}\right)$ and $f(\nu)=(1-2 \nu) /[2(1-\nu)]$ in plane strain, and $\bar{E}=E$ and $f(\nu)=(1-2 \nu) / 2$ in plane stress. The usual definition of the phase angle requires the assumption of a sharp crack, so that the stresses along an interface are of the form $[24,25]$

$$
\sigma_{y y}+i \tau_{x y}=\frac{K r^{i \epsilon}}{\sqrt{2 \pi r}}
$$

where $r$ is the distance ahead of the crack tip,

$$
\epsilon=\frac{1}{2 \pi} \ln \frac{(1-\beta)}{(1+\beta)},
$$

and $K$ is a complex stress-intensity factor, whose real and imaginary parts reduce to the well-known mode-I and mode-II stress-intensity factors in the special case of 
$\epsilon=0$. Furthermore, the crack-opening displacements are given by [24, 25]

$$
\delta_{y y}+i \delta_{x y}=\frac{8 \sqrt{1-\beta^{2}}}{1+2 i \epsilon} \frac{K}{\bar{E}^{*}} \sqrt{\frac{r}{2 \pi}} r^{i \epsilon},
$$

where $\bar{E}^{*}$ is an average modulus defined by $1 / \bar{E}^{*}=\left(1 / \bar{E}_{1}+1 / \bar{E}_{2}\right) / 2$. The $r^{i \epsilon}$ factor indicates that the displacements oscillate near the crack tip.

The phase angle is defined in terms of the ratio between the imaginary and real parts of the stress-intensity factor. This is often identified as a measure of the ratio between the shear and normal stresses, or as a ratio between the shear and normal displacements. However, Eqns. 3 and 5 indicate that this ratio varies along the interface when $\beta \neq 0$. Under these conditions, the definition of the phase angle needs to include a length scale. In a layered material, the layer thickness, $h_{1}$ (Fig. 1), is the most convenient length scale to use, and a general definition of the "nominal" phase angle becomes

$$
\psi^{\infty}=\arctan \frac{\Im\left(K h_{1}{ }^{i \epsilon}\right)}{\Re\left(K h_{1}{ }^{i \epsilon}\right)} .
$$

The choice of layer thickness as the length scale obviously divorces the definition from the facture process occurring at the crack tip, and emphasizes that this is the definition of a "nominal" phase angle when $\beta \neq 0$. However, even when $\beta=0$ and a length scale is not needed to define the phase angle by LEFM, significant discrepancies can occur between the nominal phase angle and the actual phase angle that might describe conditions at the crack tip. For example, the relationship between the two phase angles is governed by issues such as a modulus mismatch between the interphase and substrate [26], the precise location of a crack within an interphase or adhesive layer [27] or next to an interface [28], or the orientation of the crack tip relative to the interface $[29,18]$.

When using interfacial fracture mechanics for design purposes, the normal practice is to produce a curve of nominal toughness against nominal phase angle, and then to assume that this relationship can be used as a general design criterion for the interface in other geometries. Obviously, in the ideal case of a crack-tip stress field being embedded within a singular field, the approach would seem to be rigorously valid, as the local conditions at the crack tip would always be controlled by the singular field. While many experimental studies on mixed-mode fracture limit themselves to measuring how the toughness varies with nominal phase angle, a few studies do indicate that there are conditions under which nominal mixed-mode fracture properties can indeed be used in a predictive fashion [30,31,32]. However, the relative paucity of these types of studies leave open the question of how universally useful this design approach is, and whether it becomes limited for tough interfaces when the crack-tip stress field may not be embedded in a singular elastic field. Given the difficulty of 
doing experiments in this area, a key component of this paper is a set of numerical experiments that examine this question and show how mixed-mode fracture can be accommodated within an intellectual framework that recognizes the reality and role of finite cohesive strengths on the fracture of interfaces. The philosophy behind the work presented in this paper is that the cohesive-zone analyses provide what are essentially numerical experiments conducted on systems with known interface properties. Of particular interest will be the extent to which LEFM analyses can be used to predict the results of these numerical experiments, following the identical approaches that would be used to apply LEFM techniques to predict and analyze "real" experiments - namely, if a nominal energy-release rate and phase angle are computed for a particular geometry, and if they are combined with an appropriate mixed-mode failure criterion, will the correct strength be predicted?

\subsection{Issues related to the use of cohesive-zone models}

Cohesive-zone models provide a coherent analytical framework for fracture along interfaces that naturally incorporates both strength and energy criteria [33, 34]. By incorporating traction-separation laws in a finite-element model, the physical basis of interfacial fracture can be captured within a numerical analysis. In particular, the physical requirement for a dual failure criterion of exceeding both a critical stress level and a critical energy density at the crack tip is naturally met with this approach.

The concept of cohesive-zone modeling came from the original work of Dugdale [35] and Barenblatt [36], where tractions were introduced across a crack surface to remove the singularity at a crack tip in an elastic body. If the cohesive tractions are embed-

ded along a finite length within a perfectly elastic material, the length of region over which cohesive tractions are applied is prescribed by the requirement to remove the singularity [37], and the approach becomes a numerical tool rather than an attempt to connect fracture to physical phenomena. The cohesive-zone approach can be made considerably more powerful by taking the traction-separation law to represent the physical bonding across an interface or the deformation of an interphase between two adherends. In this approach, the entire interface is represented by cohesive tractions with no need for an a priori definition of the extent of the cohesive zone. If the interface bonding derives from atomic, molecular or physical bonding, well-described, exact forms of the traction-separation law could be used. If the interface is bonded by means of an interphase, then the traction-separation law can be taken as a characteristic representative of the effective tractions exerted by the interphase on the adherends [12, 38].

There are two key questions that are addressed in this paper. (i) To what extent can interfacial fracture mechanics be used to quantify the delamination of layered 
materials when the interface has a finite strength? Of particular interest is whether the concept of a nominal phase angle, as defined by interfacial fracture mechanics, provides a useful measure of the mode-mixedness of the fracture process. (ii) How do finite cohesive strengths affect the fracture of an interface under conditions when interfacial fracture mechanics may not be valid?

These questions were considered by using a simple cohesive-zone model to describe the bonding across an interface, based on the mixed-mode formulation by Yang and Thouless [13]. The numerical simulations were run using the commercial finite element package, ABAQUS. The adherends were modeled using two-dimensional plane-strain continuum elements. The cohesive-zone was implemented as two-dimensional, plane strain elements defined with the user-defined element feature of ABAQUS using a FORTRAN subroutine. An example of the subroutine is listed in [39]. Trapezoidal laws, as shown in Fig. 2, were chosen as representative forms for both the nominal and shear stresses across the interface. The normal strength of the interface is $\hat{\sigma}$ and the shear strength of the interface is $\hat{\tau}$. The mode-I toughness (area under the normal traction-separation curve) is $\Gamma_{I}$, and the mode-II toughness (area under the shear traction-separation curve) is $\Gamma_{I I}$. While some aspects of fracture can depend on the details of the traction-separation laws used, often only the strengths and toughnesses are required to describe many of the important aspects of interfacial fracture. In particular, sensitivity analyses indicated that the major conclusions of this paper are not significantly affected by the shape of the traction-separation law assumed in this study (including the choice of the displacement ratios indicated in Fig. 2). The details of some of the results presented in this paper depend on the specific assumptions made about about the properties of the interface, but the fundamental conclusions that are emphasized in this work are not.

Since two independent traction-separation laws have been used for normal and opening displacements, they need to be linked by a mixed-mode failure criterion. The criterion used in this study is given by [13]

$$
\frac{\mathcal{G}_{I}}{\Gamma_{I}}+\frac{\mathcal{G}_{I I}}{\Gamma_{I I}}=1
$$

where $\mathcal{G}_{I}$ is the mode-I energy-release rate (or mode-I energy density at the crack tip), $\mathcal{G}_{I I}$ is the mode-II energy-release rate (or mode-II energy density at the crack tip), and the energy density is defined as the area under the appropriate traction-separation law at any instant and location on the interface of interest (Fig. 2). This failure criterion is a simple one that appears to work well in predicting experimental results [13]. However, related calculations conducted as part of this investigation showed that the fundamental results presented in this paper are not sensitive to the precise form of the mixed-mode failure criterion. Other forms, such as non-linear combinations of the contributions from the two modes, give essentially similar conclusions to those that 
will be presented here. This failure criterion allows a wide variety of different types of interfaces to be considered. Interfaces that fail by what we term "mode-independent" failure, in which the shear and normal energies contribute equally to fracture, can be modeled by setting $\Gamma_{I I} / \Gamma_{I}=1$. An interface that fails only in response to the mode-I loading can be modeled by setting $\Gamma_{I I} / \Gamma_{I} \gg 1$. An extreme example of the latter case might be an interface that slips, rather than fractures, in response to shear loading. For such an interface, the magnitude of the shear strength could be considered to represent the frictional stress (for a constant friction assumption), or the flow stress of a ductile interphase. For example, a lubricated interface (with a liquid interphase), could be modeled by a very low values of $\hat{\tau} / \hat{\sigma}$ coupled with a very high value of $\Gamma_{I I} / \Gamma_{I}$.

As discussed in the previous section, interfacial fracture mechanics incorporates mixed-mode fracture by assuming that the toughness of an interface can be expressed as a function of the nominal phase angle, which is defined in terms of the stressintensity factors, as indicated in Eqn. 6. However, an alternative definition of modemixedness is needed when the concept of finite cohesive stresses is introduced. Alternative definitions in terms of stress or displacement ratios are not generally useful, as these ratios are often liable to be simple measures of the shear and normal cohesive parameters, since both the critical crack-tip displacements and stress levels at the crack tip are fixed by the fracture properties of the interface. An alternative definition of the phase angle, that makes an immediate connection to LEFM concepts and that will be shown to be very powerful within a cohesive-zone context, is to regard it as a measure of how the energy density on the interface is partitioned into shear and normal components. The definition of the phase angle then becomes [13]

$$
\psi=\tan ^{-1}\left(\sqrt{\mathcal{G}_{I I} / \mathcal{G}_{I}}\right)
$$

with $\mathcal{G}_{I I}$ and $\mathcal{G}_{I}$ defined earlier as the area under the traction-separation laws at the point of interest. If $\mathcal{G}_{I I}$ and $\mathcal{G}_{I}$ are taken to be the nominal values of the mode-I and mode-II energy-release rates (as defined from an LEFM analysis for $\beta=0$ ), the nominal phase angle, $\psi^{\infty}$, results from the definition of Eqn. 8. This nominal phase angle can then be used in conjunction with the mixed-mode failure criterion of Eq. 7, to determine how the mixed-mode toughness varies as a function of nominal phase angle:

$$
\Gamma=\Gamma_{I} \frac{\lambda\left(1+\tan ^{2} \psi^{\infty}\right)}{\lambda+\tan ^{2} \psi^{\infty}}
$$

where $\lambda=\Gamma_{I I} / \Gamma_{I}$. The form of this equation is very similar to various forms of a mixed-mode failure criteria that have been used in interfacial fracture mechanics to analyze mixed-mode phenomena [11], and is used as the LEFM mixed-mode failure criterion in this paper.

In a cohesive-zone analysis, Eqn. 8 can be used to calculate the phase angle at any point along the interface, since $\mathcal{G}_{I I}$ and $\mathcal{G}_{I}$ can be determined at any node of the 
user-defined elements describing the cohesive law along the interface. In particular, it is useful to define a "crack-tip phase angle", $\psi_{o}$, which is the phase angle calculated at the first node of the cohesive zone ahead of the crack tip. It is recognized that, in the absence of the singular stress field that is associated with cracks in linearelasticity, the definition of a crack tip is somewhat arbitrary. Those who think of cohesive tractions across an interface in terms of bridging will associate the crack tip with a different point from those who think in terms of damage [40]. In this paper, the crack tip is defined as being located at the first node along the interface that supports no tractions. However, it will be demonstrated that the reported values of the crack-tip phase angle represent the mode-mixedness of the interface over some extent from the crack tip. In addition, mesh sensitivity analyses have demonstrated that the crack-tip phase angle, as calculated in this paper, has physical significance, and is not mesh-dependent.

\subsection{Mode-I fracture with a cohesive zone}

Some of the issues associated with a cohesive-zone model of fracture, and how it provides a smooth transition between an Inglis and a Griffith approach to fracture, can be demonstrated by a simple mode-I geometry. For these purposes, a simple two-dimensional tensile bar subjected to a uniaxial load with a central crack aligned perpendicular to the sides of the bar, and a fracture plane extending from the crack tip to the specimen boundaries can be used. Since the geometry is purely mode-I, the fracture load, $P_{f}$ is a function of

$$
\bar{E}, a, L, \Gamma_{I}, \hat{\sigma}
$$

where $\bar{E}$ is the modulus of the bar, $2 a$ is the crack length, $L$ is the length of the ligament from each crack tip to the free surface, $\Gamma_{I}$ is the mode-I toughness and $\hat{\sigma}$ is the normal cohesive strength. From standard LEFM solutions [41], the fracture load predicted by LEFM depends only on the geometry, modulus, and the interfacial toughness. It is given by

$$
P_{L E F M}=\sqrt{\frac{4 \bar{E} \Gamma_{I} a}{\pi}} f(a / L),
$$

where,

$$
f(a / L)=\frac{(1+a / L)^{5 / 2}}{0.826(a / L)^{3}+1.5(a / L)^{2}+(a / L)} .
$$

A strength-based failure criterion would predict that fracture occurs when the average stress supported by the ligaments is equal to the cohesive strength of the interface. In 
other words, the failure load depends only on the geometry and the cohesive strength. It is given by

$$
P_{\text {strength }}=2 L \hat{\sigma}
$$

Figure 3(a) shows how the strength for this mode-I geometry varies with fracturelength scale. The strength is normalized in two different ways. One plot shows the ratio of the fracture load to the fracture load that would be predicted using toughness only as a fracture parameter, $P_{f} / P_{L E F M}$. The other plot shows the ratio of the fracture load to the fracture load that would be predicted using strength only as a fracture parameter, $P_{f} / P_{\text {strength}}$. For the purposes of comparison with these two ratios, plots of $P_{L E F M} / P_{\text {strength }}$ and $P_{\text {strength }} / P_{L E F M}$ are superimposed on this figure. This figure makes it very clear how a cohesive-zone model permits a smooth transition between a strength-based failure criterion and an energy-based failure criterion. Both criteria provide upper bounds to the actual fracture load and, as would be expected, the lowest upper bound dominates the failure mechanism. At low values of the fracture-length scale, the toughness criterion for fracture dominates, and LEFM provides an excellent model for fracture. At higher values of the fracture-length scale, the strength criterion dominates, and strength models provide excellent predictions for the maximum load that can be supported before fracture.

The observations of the previous paragraph would suggest that the tractions along the interface should tend to two limits. At very low fracture-length scales, where an energy-balance can be used to predict the strength, the tractions along the interface should follow the the inverse-square-root dependence on distance from the crack tip, $r$ which is given by

$$
\sigma=\sqrt{\bar{E} \Gamma_{I} /(2 \pi r)}
$$

With a cohesive zone at the crack tip, this singular field would be expected to exist only in an intermediate region. It would encompass a crack-tip region where the stresses are limited by the cohesive strength of the interface. Remote from the crack tip, the tractions would tend to the far-field distribution. At large fracture-length scales, where a force-balance can be used to predict the strength, the stresses should be uniform along the interface. These concepts can be explored by examining how the tractions resulting from the cohesive elements vary along the interface. It can be seen from Fig. 3(b) that the two limiting behaviors are observed as expected. A very small fracture-length scale of $\bar{E} \Gamma_{I} / \hat{\sigma}^{2} a=0.055$ means that fracture is accurately predicted by the LEFM solution. Under these conditions, there is a limited region of the interface, between $r / L=0.06$ and $r / L=0.12$, over which the tractions follow an inverse-square-root singular form. At very large fracture-length scales, the tractions along the interface are essentially uniform. It is in this regime that the strength of the cracked body is determined only by the cohesive strength of the interface. These observations are consistent with earlier descriptions of how fracture-length scales are associated with flaw-tolerant behavior and flaw-sensitive behavior [42]. 


\subsection{Mixed-mode fracture with a cohesive zone}

The effects of mixed-mode loading can be explored with the same center-cracked geometry used in the previous section, but with the addition of a shear force per unit width, $Q$, in addition to the normal force, $P$. Both the phase angle and energy-release rate for this mixed-mode geometry can be computed using a continuum finite-element calculation. With $Q / P=1, a / L=0.9, H / L=20$ and $H_{o} / L=7.4$, the energy-release is

$$
\mathcal{G}=(0.169 \pm 0.001) P^{2} / \bar{E} a,
$$

and the phase angle is

$$
\psi^{\infty}=46.0 \pm 0.5^{\circ} .
$$

For a mode-independent failure criterion, the fracture load predicted by LEFM, $P_{L E F M}$, is given by equating $\mathcal{G}$ in Eqn. 13 to the mode-I toughness $\Gamma_{I}$.

The cohesive strengths are parameters in a cohesive-zone analysis, so that the failure load, $P_{f}$, normalized by the mode-I toughness, and the crack-tip phase angle, $\psi_{o}$, for this geometry both depend on the following non-dimensional parameters:

$$
\frac{\Gamma_{I}}{\bar{E} a}, \frac{\bar{E} \Gamma_{I}}{\hat{\sigma}^{2} a}, \frac{Q}{P}, \frac{a}{l}, \frac{H}{L}, \frac{H_{o}}{L}, \frac{\Gamma_{I I}}{\Gamma_{I}}, \frac{\hat{\tau}}{\hat{\sigma}} .
$$

Figure 4(a) shows how the strength varies with the fracture-length scale, $\bar{E} \Gamma_{I} / \hat{\sigma}^{2} a$ for mode-indpendent failure $\left(\Gamma_{I I} / \Gamma_{I}=1\right)$. It will be observed that the LEFM calculations give excellent predictions (to within about $95 \%$ ) for the results of the numerical experiments, provided the fracture-length scale is less than about 1 . The predicted strength is accurate to within $98 \%$ for fracture-length scales of $\bar{E} \Gamma_{I} / \hat{\sigma}^{2} a<0.4$ (which corresponds to the conventional fracture-length scale taken to indicate the validity of LEFM). Figure 4(b) indicates that the crack-tip phase angle (calculated from the node immediately ahead of the crack tip) is identical within numerical uncertainties to the nominal phase angle predicted by LEFM at small fracture-length length scales. The agreement between the two phase angles is within $5 \%$ at fracture-length scales up to about 3. This agreement is significant, because it means that one should expect LEFM predictions of strength to be accurate for mode-dependent failure criteria in

this range of fracture-length scales. Furthermore, this agreement suggests that it is appropriate to regard the nominal phase angle as a measure of how the shear and normal energy-release rates along the interface are related, rather than as a measure of displacement or stress ratios that must be sensitive to definitions of interfacial tractions. These points will be revisited in more detail in the subsequent sections. 
As in the previous section, the tractions along the interface can be extracted from the results of the numerical experiments. It should be noted again, that in these calculations, cohesive elements were placed along the entire length of the ligament, from the crack tip to the free surfaces. There were no continuum elements along the interface, so the tractions that arise are dictated only by the interaction of the geometry and the traction-separation law. Figure 5 shows how the normal and shear tractions at fracture vary along the interface for different values of the fracture-length scale. Superimposed on these plots are lines corresponding to the singular tractions predicted from LEFM. When the fracture-length scale is very small, the stress distribution exhibits the expected intermediate region in which the interfacial tractions follow an inverse-square-root law. As in the earlier example, the tractions in this region are slightly higher than those expected from linear-elasticity, because the tractions are limited to the cohesive strengths at the crack tip. At very large values of the fracturelength scale, where one might expect the failure load to be determined by the cohesive strength of the interface, rather than by the interfacial toughness, the tractions along the ligament are essentially uniform. A fracture length scale of $\bar{E} \Gamma_{I} / \hat{\sigma}^{2} a=1$ appears to be close to the point above which the stresses do not exhibit any inverse-squareroot characteristics. However, it is interesting to note from Fig. 4(b) that the nominal phase angle still provides a good prediction for the mode-mixedness of the crack tip, even at fracture-length scales between 1 and 3, when the tractions (particularly the shear component) don't really exhibit any inverse-square-root behavior.

\section{Numerical results for beam-like geometries}

Beam-like geometries (Fig. 1) were chosen to form the major focus of this study. Becker et al. [43] explored the elastic crack-tip stress field for these geometries, and concluded that the extent of the singular field was limited to less than about one hundredth of the layer thickness. This is so small that concerns were raised about the appropriateness of linear-elastic fracture mechanics to describe these geometries. These issues are addressed in the next four sections. In the first section, modeindependent fracture is considered. In the following section, mode-dependent fracture is considered. In the third section, issues associated with fracture under a compressive interfacial stress are addressed. Then in the final section, the effects of modulus mismatch are addressed very briefly.

For beam-like geometries being analyzed by a cohesive-zone analysis, the nondimensional failure loads and crack-tip phase angles are dependent on the following dimensionless groups:

$$
\frac{\Gamma_{I}}{\bar{E}_{1} h_{1}}, \frac{\bar{E}_{1} \Gamma_{I}}{\hat{\sigma}^{2} h_{1}}, \frac{a}{h_{1}}, \frac{L}{h_{1}}, \frac{h_{1}}{h_{2}} \cdot \frac{\Gamma_{I I}}{\Gamma_{I}}, \frac{\hat{\tau}}{\hat{\sigma}}, \alpha, \beta .
$$

In general, other dimensionless groups associated with issues such the shape of the 
cohesive law and the failure criterion would enter this list. The details of some of the results that are presented in the following sections can be affected by the values chosen for these parameters. However, as discussed earlier, sensitivity analyses showed that the essential results and trends that have been high-lighted as being the major conclusions of this study were not affected. In a similar vein, the effects of mesh-dependency were also explored. The results presented are mesh independent to within what was considered to be an acceptable range of numerical uncertainty (indicated by error bars on the accompanying plots).

\subsection{Mode-independent fracture}

As discussed earlier, the crack-tip stress field makes no contribution to the energyrelease rate for the steady-state configuration of a laminated geometry subjected to a combination of pure moments and axial loads (Fig. 1a). Therefore, under these conditions, the energy-release rate must be valid no matter how large the fracturelength scale is. The only requirement on the geometry required for a steady-state configuration is that the crack and ligament must be long enough to ensure a region of a uniform stress state ahead and behind the crack tip. If these conditions are met, the details of the cohesive law have no influence on the energy-release rate. Then, if the interface toughness is mode-independent, the conditions for crack growth will be rigorously defined by equating the energy-release rate to the toughness for all fracture-length scales.

A different situation arises if a laminated geometry is loaded by a transverse force (Fig. 1b). The energy-release rate for this loading condition cannot be determined from a steady-state energy-balance, and the details of the crack-tip stress field are always important [15]. Therefore, the strength (and other characteristics) of the interface can affect crack growth. However, the effects of such a transverse load can often be hidden because, in practice, the shear load gives rise to a moment acting at the crack tip. This moment can often dominate the driving force for fracture. The work of $\mathrm{Li}$ et al. [15] gives insight into the effects of a transverse shear and its associated moment. For a homogeneous double-cantilever beam, with a crack of length $a$ and $h_{2}=h_{1}$, the critical transverse shear force required for fracture is given by

$$
\frac{F_{f}}{\Gamma_{I}}=0.289 \sqrt{\frac{\bar{E} h_{1}}{\Gamma_{I}}}\left(\frac{a}{h_{1}}+0.674\right)^{-1} .
$$

The first term in the bracket corresponds to the result for a pure moment, while the second term in the bracket corresponds to the shear term. (In this special symmetrical case only, the two terms add. In general, they don't add in this fashion [15]). An analytical result for the effects of a triangular cohesive zone was also derived for this 
geometry. ${ }^{2}$ The fracture load in the presence of a triangular cohesive law is given by

$$
\frac{F_{f}}{\Gamma_{I}}=0.289 \sqrt{\frac{\bar{E} h_{1}}{\Gamma_{I}}}\left[\frac{a}{h_{1}}+0.674\left(1+1.62 \frac{\bar{E} \Gamma_{I}}{\hat{\sigma}^{2} h_{1}}\right)^{1 / 4}\right]^{-1} .
$$

As expected from the discussion above, the finite strength of the interface affects the shear term, but not the moment term. As a result, it can be seen from this equation that when the moment term is directly associated with a transverse shear load term, the effect of the cohesive zone depends on how the fourth root of $\bar{E} \Gamma_{I} / \hat{\sigma}^{2} h_{1}$ compares to the normalized crack length $a / h_{1}$. This general form of relationship will also be apparent from the results of the numerical analysis that are presented next.

The concepts discussed above are illustrated by numerical experiments for an asymmetric double-cantilever beam of the form shown in Fig. 1b, loaded by a transverse shear force, and using the cohesive law of Fig. 2. For the calculations of this section, a homogeneous geometry, with $\bar{E}_{1}=\bar{E}_{2}=\bar{E}$, and mode-independent cohesive properties, $\hat{\tau} / \hat{\sigma}=1$ and $\Gamma_{I I} / \Gamma_{I}=1$, were assumed. Figure 6(a) shows how the failure load depends on both the ligament size and the fracture-length scale, for a crack length of $a / h_{1}=20$. Superimposed on this plot is the steady-state LEFM prediction for the fracture load appropriate for long ligaments, calculated from the results of Refs. [14] and [15]. Separate continuum finite-element calculations were used to compute mode-independent LEFM predictions for the failure load away from steady-state, for small ligaments. These were essentially indistinguishable from the cohesive-zone results shown in Fig. 6(a) for a fracture length scale of $\bar{E} \Gamma_{I} / \hat{\sigma}^{2} h_{1}=1.43$. Even when the fracture-length scale is as large as 100, the LEFM prediction is correct to within $5 \%$. However, the relative error associated with a fracture-length scale this large becomes more significant away from steady-state, as the ligament size decreases. The dramatic increase in the accuracy of LEFM predictions expected as the crack-tip loading becomes increasingly dominated by bending is shown in Fig. 6(b), in which $a / h_{1}=400$. Now, it can be seen that the mode-independent LEFM predictions are accurate for fracture-length scales greater than about $\bar{E} \Gamma_{I} / \hat{\sigma}^{2} h_{1}=10^{5}$. As indicated in the previous paragraph, mode-independent fracture is not affected by the cohesive strength of the interface in steady-state geometries. A compliant interface, associated with a large fracture-length scale, results in a lower failure load only when steadystate solutions are not applicable.

\footnotetext{
${ }^{2}$ There is a typographical error in Eqn. 20 of Ref. [15]. It should be corrected to read $k=$ $[1.615(\bar{E} / h)]\left[1+1.615\left(\bar{E} \Gamma_{I o} / \hat{\sigma}^{2} h\right)\right]^{-1}$. The other equations appear to be correct.
} 


\subsection{Mode-dependent fracture}

\subsubsection{Effects of mode-II toughness}

The existence of steady-state solutions for the energy-release rate gave a strong basis for expecting that mode-independent delamination of beam-like geometries is relatively insensitive to any properties of the interface other than toughness. However, the energy-balance arguments that underpin the steady-state solutions provide no information about phase angles, and it is clear from experiments that mixed-mode effects play a key role in many delamination phenomena. For example, the finite widths of buckle-driven delaminations [11,30] and crack-front instabilities [44, 45] are dependent on a mode-dependent toughness. At the heart of the analyses of these phenomena are calculations of a nominal phase angle based on the assumptions of linear-elasticity and the use of stress-intensity factors. Therefore, there might be concerns as to whether these solutions should be valid, except under very restrictive conditions for which the cohesive stresses are embedded within an inverse-square-root field [43]. To explore this issue, the cohesive-zone analyses are extended to examine how mixed-mode fracture is affected by mode-dependent cohesive properties of the interface. In the results that follow, LEFM predictions based on the mixed-mode failure criterion of Eqn. 9 are compared to the failure loads obtained from the numerical experiments, so as to investigate how the accuracy of the predictions depends on the fracture-length scale. In addition, crack-tip phase angles, $\psi_{o}$, are compared to the nominal phase angles, $\psi^{\infty}$, calculated from LEFM analyses.

Figures $7(\mathrm{a})$ and (b) show how the failure loads obtained from the numerical experiments for two different loading configurations depend on the fracture-length scale for different ratios of $\Gamma_{I I} / \Gamma_{I}$. Figure 7 (a) shows the results for a homogeneous, asymmetrical double-cantilever beam loaded only by a transverse shear load. Figure 7(b) shows the same type of specimen, loaded by a transverse shear load and a fixed axial load. Superimposed on these plots are the associated predictions for the failure load calculated from LEFM, using the mixed-mode failure criterion of Eqn. 9 and based on the nominal phase angles obtained from Refs. [14] and [15]. As can be seen, these LEFM results provide excellent predictions for mixed-mode failure over a significant range of fracture-length scales. (In this context, it should be noted that the appropriate non-dimensional group to define the fracture-length scale changes to one containing $\Gamma_{I I}$, rather than $\Gamma_{I}$, when $\Gamma_{I I}>\Gamma_{I}$.) These results indicate that mixed-mode LEFM analyses, based on nominal phase angles, are useful for predictive purposes over ranges of fracture-length scales that would seem to include many practical applications such as tough adhesive joints, provided no plasticity occurs in the adherends.

The results of Fig. 7 indicate that the nominal phase angle provides information about the mode-mixedness at the crack tip. This concept is explored in Figs. 8(a) 
and (b), in which the crack-tip phase angle at the point of crack advance, computed from Eqn. 8 for the first node ahead of the crack tip, is plotted as a function of fracture-length scale for the two geometries considered in the previous paragraph. Superimposed on these plots are the nominal phase angles calculated from LEFM. In the specimen loaded by a transverse shear load and a fixed axial load, the nominal phase angle at fracture is dependent on $\Gamma_{I I} / \Gamma_{I}$, because this ratio determines the failure load. It will be noted from these plots that the phase angle at the crack tip and the nominal phase angle are essentially identical at the smaller length scales, and are in excellent agreement over the range for which the failure loads are correctly predicted. Once again, it should be noted that this identity between the nominal phase angle and the crack-tip phase angle indicates that the nominal phase angle is best thought of as a relative measure of the shear and normal energy-release rates, rather than as a ratio of stresses or displacements.

Figure 9(a) shows how the normal and shear tractions vary as a function of distance along the interface ahead of the crack tip for a non-dimensional fracture-length scale of $\bar{E} \Gamma_{I} / \hat{\sigma}^{2} h_{1}=1.43$. Superimposed on this plot are lines corresponding to the normal and shear tractions associated with the inverse-square-root field of linear elasticity. As can be seen from Fig. 8(a), the actual phase angle is in excellent agreement with the nominal phase angle under these conditions. However, Fig. 9(a) shows that there is no part of the stress field, either for the shear or normal stresses, that can be identified as following the inverse-square-root dependence associated with a stress-intensity factor at this fracture-length scale. This complete divorce from any singular field is much more apparent in this beam-like geometry than it was for the central-crack problem considered earlier. These results indicate very dramatically that the nominal phase angles based on LEFM calculations can accurately describe mixed-mode fracture of beam-like geometries, and there appears to be no requirement for the cohesive zone to be embedded within a linear-elastic singular field. This is a surprising but extremely important result implying a very general utility of the interfacial fracture mechanics results for these geometries.

The physical significance of the crack-tip phase angle as defined earlier for cohesive models is demonstrated in Fig. 9(b). In this plot, the phase angle is calculated from Eqn. 8 for a succession of nodes in the cohesive zone away from the crack tip. It will be seen that the phase angle asymptotes to the crack-tip phase angle at a distance of about $r / h_{1}=0.01$ from the crack tip, which is two orders of magnitude larger than the size of the elements used to describe the cohesive zone at the crack tip. Within experimental uncertainty, this crack-tip phase angle is identical to the nominal phase angle obtained from LEFM solutions. 


\subsubsection{Effects of interfacial shear strength}

There are many types of interfaces that can support normal stresses quite readily, but are weak in shear. Examples include lubricated interfaces, interfaces between MEMS components that are susceptible to stiction, and interfaces formed by electrostatic, magnetic or other physical bonds. The normal cohesive strength of the interface is generally associated with the bonding, but the shear strength corresponds to a flow stress or to a frictional resistance of the interface. The response of these slipping interfaces can be modeled using a cohesive-zone approach. The results of calculations to explore what happens as the shear strength of the interface is decreased and the mode-II toughness is increased ${ }^{3}$ are presented in Fig. 10 for an asymmetrical-doublecantilever beam geometry loaded by a transverse load. Figure 10(a) shows how the failure load for a system with such an interface can vary with the ratio of the shear to normal strength. Figure 10(b) shows how the crack-tip phase angle depends on this ratio. Superimposed on these two plots are the nominal phase angle, and the predicted failure loads based on this nominal phase angle. In these plots, the nondimensional fracture-length scale based on mode-I parameters is only 0.143 , which is sufficiently small that LEFM predictions are expected to be accurate. However, the mode-II parameters are allowed to vary over a range that moves the failure load and the phase angle away from the LEFM predictions, when $\hat{\tau} / \hat{\sigma}<1$. As the shear strength drops, the magnitude of the crack-tip phase angle drops to zero; i.e., the crack tip tends to a state of pure mode-I. Associated with this decrease in phase angle, the failure loads all tend to the mode-independent value.

These results are in agreement with experimental observations of mixed-mode fracture of a lubricated interface reported by Thouless [10]. In those experiments a bonded interface between two glass plates was formed in an asymmetrical-double-cantilever beam geometry using the capillary force exerted by a thin film of diffusion-pump oil. The specimen was loaded by means of a wedge, and the toughness of the interface was computed as a function of the nominal phase angle (based on LEFM calculations). It was found that the toughness was independent of the nominal phase angle and equal to the change in surface energy associated with de-wetting the glass/oil interface. It was also noted that the usual implication of such a phase-angle independent toughness would be that the energy dissipated by fracture under mode-II deformation should be equal to the energy dissipated by fracture under mode-I deformation. However, it was noted that such an interpretation would be paradoxical for such a system in which slip, not fracture, is expected to occur in response to mode-II deformation. It was conjectured that the results demonstrated the difference between a nominal and crack-tip phase angle. The results of Fig. 10 show clearly that in a system with a lubricated interface, the deformation at the crack tip is essentially pure mode-I, and

\footnotetext{
${ }^{3}$ At a simple level, one might envision the limit of an interface that can slip but not fracture in mode-II, as being an interface with an infinite value of $\Gamma_{I I}$ and a finite value of $\hat{\tau}$.
} 
that a mixed-mode fracture experiment of the sort described in Ref. [10] would indeed result in a nominal toughness that was independent of the nominal phase angle. ${ }^{4}$

\subsection{Compressive stresses at crack tips}

Fracture under conditions in which the crack tip is closed by compressive stresses is of significance in a number of delamination phenomena. For example, it occurs in the edge delamination of layers with residual compression if the edge defect is too small to permit buckling [31]. It also accompanies buckling-driven delamination once the buckle reaches a critical size $[11,30,46]$. For example, in experimental studies using mica bonded to aluminum [30], it was shown that delamination can occur under conditions associated with negative nominal mode-I stress-intensity factors (nominal phase angles with a magnitude greater than $90^{\circ}$ ). In Ref. [30], experimental observations for buckling-driven delamination were used to compute a nominal toughness as a function of nominal phase angle. In that paper, the resulting plots were terminated when the nominal phase angle reached $-90^{\circ}$. However, using the data provided in Ref. [30], the plots can be extended up to a nominal phase angle of almost $-130^{\circ}$. In the classical approach of linear elasticity, such nominal phase angles would correspond to interpenetration of the two crack surfaces. Since this violates physical reality, analyses of buckling-driven delamination have been limited to the assumption that contact of the crack surfaces suppresses any negative component of the mode-I stress-intensity factor, so that fracture occurs under pure mode-II conditions. This contact of the crack surfaces can lead to frictional dissipation. Models of Coulomb friction [46] and constant friction [30] have been used to calculate the extent to which the toughness may be elevated above the intrinsic mode-II value under conditions of crack-tip contact, with particular application to buckling-driven delamination.

Interfacial fracture mechanics models do not incorporate the concept of a finite interfacial thickness associated with the equilibrium separation between two adherends. There will always be a finite thickness at some scale, whether the bonding plane is limited to a single atomic bond or to a more macroscopic interphase. Cohesive-zone models allow this physical concept to be incorporated. Furthermore, an interface that has a finite thickness can accommodate some negative mode-I deformation before contact of the crack surfaces occurs. This means that the notion of a phase angle with a magnitude greater than $90^{\circ}$ can have physical significance. Within this view of an interface, it seems reasonable to assume that negative mode-I deformations do not contribute to failure. Therefore, the compressive mode-I cohesive energy is stored, and then lost when the condition $\mathcal{G}_{I I}=\Gamma_{I I}$ is met. The failure criterion of Eqn. 7

\footnotetext{
${ }^{4}$ The asymmetric double-cantilever-beam geometry doesn't exhibit large-scale slip when the interface is weak in shear. If axial loads were applied to this geometry, large-scale slip would occur. If LEFM were used to analyze the geometry, the nominal toughness would increase.
} 
can then be modified to the following form:

$$
\begin{gathered}
\frac{\Gamma}{\Gamma_{I}}=\frac{\lambda\left(1+\tan ^{2} \psi^{\infty}\right)}{\lambda+\tan ^{2} \psi^{\infty}}, \quad\left|\psi^{\infty}\right| \leq 90^{\circ} \\
\frac{\Gamma}{\Gamma_{I}}=\frac{\lambda\left(1+\tan ^{2} \psi^{\infty}\right)}{\tan ^{2} \psi^{\infty}}, \quad\left|\psi^{\infty}\right| \geq 90^{\circ} .
\end{gathered}
$$

A limit on the magnitude of the nominal phase angle depends on the thickness of the interface, as it is set by any crack-surface contact that might occur.

To investigate the issues associated with compressive stresses across the interface, a set of numerical experiments were conducted on an asymmetrical-double cantileverbeam geometry with fixed cohesive properties. The specimen was clamped at one end, and loaded by various combinations of applied axial load, $P$, and transverse load, F, until failure occurred. For these specimens, contact elements with Coulomb friction were used along the crack surfaces to prevent interpenetration. Two of the specimens had relatively low values for the equilibrium separation of the interface, $t$, so that $t / h_{1}=0.00174$. One of these specimens had a coefficient of friction along the crack surfaces given by $\mu=0$; the other had a coefficient of friction given by $\mu=0.5$. The third specimen had a much thicker interface thickness of $t / h_{1}=0.0174$, and $\mu=0 . .^{5}$

Having prescribed the interface properties and frictional characteristics, the numerical experiments were conducted in an exactly analogous fashion to how physical experiments would be conducted to deduce the mixed-mode failure criterion of an interface. The axial and transverse loads were varied independently, and the magnitudes of the loads required for fracture were determined (Fig. 11a). The values of these loads were then used to calculate the appropriate crack-tip moments, axial loads and transverse shear loads for the fundamental LEFM problems [14, 15]. Once these had been determined, the nominal energy-release rate at fracture and the associated nominal phase angle were computed. The resultant plot of the nominal energy-release rate versus nominal phase angle at fracture (Fig. 11b) is then analogous to an experimentally determined mixed-mode failure criterion. For the purposes of comparison, the LEFM failure criterion for the interface (which would permit interpenetration) has been superimposed on Fig. 11b. As in the earlier sections, additional insight into the physics of the problem is provided by comparing the nominal phase angle from these LEFM calculations with the crack-tip phase angle (calculated from the numerical output of the crack-tip values of $\mathcal{G}_{I}$ and $\mathcal{G}_{I I}$ at fracture). This comparison

\footnotetext{
${ }^{5}$ The displacements ahead of the crack tip were monitored to ensure that the compressive deformations never exceeded the equilibrium thickness of the interface. However, this requirement could have been accommodated by the physical approach of modifying the traction-separation law to prevent this possibility.
} 
is shown in Fig. 11c.

The results presented in Fig. 11 have been obtained for a system with a fracturelength scale small enough for LEFM to be an appropriate way of characterizing fracture, up to the point at which the magnitude of the nominal phase angle equals $90^{\circ}$. (Although, a discrepancy of a few degrees in the phase angle translates to a relatively larger discrepancy in the nominal toughness close to $90^{\circ}$.) Once compression occurs at the crack tip, the discrepancy between the nominal phase angle and the actual phase angle increases. In particular, for the thinnest interfaces $\left(t / h_{1}=0.00174\right)$, cracksurface contact immediately eliminates any significant mode-I compression, leaving the crack-tip under pure mode-II conditions (Fig. 11c). This situation, is then identical to existing LEFM analyses of the problem [30, 46]. If there are no other dissipative processes $(e . g ., \mu=0)$, the nominal toughness is identical to the mode-II toughness under these conditions. Frictional dissipation (e.g., $\mu=0.5$ ), can cause the nominal toughness to rise above this pure mode-II level. Both of these effects are very clear from the plot of Fig. 11b. If the interface is thicker, compression in the interface can be accommodated without crack-tip contact. Under these conditions phase angles greater than $90^{\circ}$ have physical significance, as shown in Fig. 11c. Accommodation of a compressive component of the mode-I energy-release rate, without it contributing to fracture, may then allow the nominal toughness to continue tracking the LEFM failure criterion of Eqn. 17 (Fig. 11b). Similar calculations to those presented here were also done for much larger fracture-length scales. The observations were very similar, with the expected drops in both the nominal toughness (for a given nominal phase angle) and the crack-tip phase angle. This latter effect delayed the onset of crack-tip contact to larger values of nominal phase angle.

\subsection{Modulus mismatch}

The final section of this paper explores the effects of modulus mismatch. There are two inherent difficulties associated with the use of LEFM to analyze fracture when the modulus-mismatch parameter $\beta$ does not equal zero. In the first place, LEFM predicts crack-surface interpenetration. It is recognized that this implies contact of the crack surfaces [47]. This certainly would be expected if the equilibrium thickness of the interphase or interface is relatively small. But, as discussed above, crack-surface contact need not occur if the interface is thick enough. A more fundamental problem is that when $\beta \neq 0$, it is not possible to partition the energy-release rate into mode-I and mode-II components. The ratio between the two modes is not fixed, but varies with distance from the crack tip. The parameters associated with LEFM do not contain enough information about the fracture process to permit the introduction of a length scale which is required to establish a phase angle when $\beta \neq 0$. While this is not an issue for mode-independent fracture, a phase angle does need to be defined for 
mode-dependent fracture. However, a major advantage of recognizing that interfaces should be described by both a strength and a toughness is that a length scale associated with the cohesive parameters is introduced naturally. As will become apparent in this section, this length scale makes the phase angle at the crack tip well defined. It is this phase angle that then controls fracture through the appropriate mixed-mode failure criterion.

LEFM solutions do not exist in the literature for loading by means of a transverse shear load with non-zero values of $\beta$. Therefore, attention in this section is restricted to a geometry that has only moments and axial loads acting at the crack tip. The geometry chosen consists of an asymmetrical double-cantilever beam, clamped at one end, and subjected to a couple formed by axial loads. Non-zero values of $\beta$ result in stress oscillations along the interface [24, 25], as can be seen in Fig. 12 which shows how the normal and shear stresses vary with distance ahead of the crack tip for a relatively small fracture-length scale. These oscillations indicate that unloading of the interface will occur during crack growth. In the present study, the cohesive elements were completely reversible. However, it will be appreciated that there are physical situations for which one might expect hysteresis (caused by damage or plasticity within the cohesive layer); the extra energy dissipation would then be expected to influence the failure criterion and result in $R$-curve behavior. This aspect of the problem has not been addressed here. While contact elements were used for the crack surfaces and the thickness of the cohesive elements was maintained at $t / h_{1}=1.736 \times 10^{-4}$, neither crack-surface contact nor inter-penetration ahead of the crack tip occurred for any of the results presented in this section. It was noted that details of the tractionseparation law such as the shape, did influence some of the details of the results presented in this section. However, the general conclusions that are emphasized are not dependent on these details. Finally, issues of mesh sensitivity were explored to ensure that the meshes used in this study were fine enough to avoid the the mesh size being a significant parameter for the results. Any effects of mesh size are within the levels of quoted uncertainty.

In Fig. 13, the normalized fracture load, $P_{f} / \Gamma_{I}$, and the crack-tip phase angle at failure, are plotted as functions of the elastic modulus mismatch. Figure 13(a), shows the results for $\beta=0$, and a range of $\alpha$; Fig. 13(b) shows the results for $\beta=\alpha / 2$, and a range of $\alpha$; Fig. 13(c) shows the results for $\alpha=0.8$ and a range of $\beta$. The results for this set of figures were calculated with a small fracture-length scale, and with mode-independent fracture properties for the interface. Under these conditions the strength is expected to be independent of $\beta$ and given by LEFM predictions, because $\beta$ does not affect the energy-release rate (for a geometry loaded by axial loads and moments), and the phase angle does not affect the fracture load (unless there is some compression at the crack tip). Figure 13 confirms that, while the crack-tip phase angle is shifted from the nominal phase angle when $\beta \neq 0$, the fracture load is not 
influenced by this shift for mode-independent cohesive properties.

Although the shift in crack-tip phase angle induced by non-zero values of $\beta$ is essentially of only academic interest for mode-independent fracture, this is not the case for mode-dependent fracture. When $\Gamma_{I I} / \Gamma_{I} \neq 1$, a shift in the crack-tip phase angle from its nominal value affects the strength of the interface. This is illustrated in Fig. 14 which shows the effect of $\beta$ on the nominal phase angle, the crack-tip phase angle, the LEFM predictions for strength based on $\psi^{\infty}$, and the strength calculated by the cohesive-zone model. The systematic discrepancy between the LEFM predictions for strength and the cohesive-zone results arises because of the absence of any information that permits the appropriate shift between the nominal phase angle and the crack-tip phase angle to be incorporated into the LEFM predictions. At negative values of $\beta$, the crack-tip phase angle is lower than the nominal value, so the strength decreases. At positive values of $\beta$, the crack-tip phase angle is higher than the nominal value, so the strength increases. If the phase shift were known, the fracture load could be correctly predicted from the LEFM analysis, when the fracture-length scale is small. Unfortunately, this information is not contained within a pure LEFM analysis; it has to be calculated from the cohesive-zone analysis.

It will have been observed from Figs. 13 and 14 that the shift in phase angle is approximately proportional to $\beta$. This is of significance, because the phase angle depends on a length scale, $\xi$, such that $[24,48]$

$$
\psi=\psi^{\infty}+\epsilon \ln \left(\xi / h_{1}\right)
$$

If the appropriate length scale for describing the shift in phase angle is independent of modulus mismatch, a plot of the difference between the crack-tip phase angle, $\psi_{o}$, and the nominal phase angle, $\psi^{\infty}$, against $\epsilon$ should give a straight line with a slope of $\ln \xi / h_{1}$. The length scale for describing the shift in phase angle can be determined from this slope. Such a plot of the data from the preceding two figures is given in Fig. 15. From the slope, the length scale is given by $\xi / h_{1}=0.0018 \pm 0.0004$ for the cohesive model used here. This length does not appear to be obviously related to some well-defined aspect of the cohesive-zone, although it does appear to be well within the range that might be considered to be part of the cohesive zone from Fig. 12.

Figure 16 shows how the phase angle (defined by Eqn. 8) varies with distance from the crack-tip, $r$, for $\alpha=0.4$, and $\beta=0.0$ and 0.2 . It can be seen that the phase angle plateaus at a value of $\psi_{o}$ in the vicinity of the crack tip. For this figure, the fracture-length scale is relatively small, and further from the crack tip is a region in which the phase angle appears to match the LEFM value. When $\beta=0$, this is represented by a second plateau given by $\psi=\psi^{\infty}$. When $\beta \neq 0$, the LEFM result corresponds to a straight line given by

$$
\psi=\psi^{\infty}+\epsilon \ln \left(r / h_{1}\right)
$$


This contrasts with the results of Fig. 9(b) in which there was only a single plateau near the crack tip, because the fracture length scale was relatively large. From the present figure it appears that, when the fracture-length scale is relatively small, there are four regimes for the phase angle: (i) a plateau near the crack tip corresponding to $\psi^{o}$; (ii) a transition region at the boundary of the cohesive zone; (iii) an intermediate region where the phase angle agrees with the LEFM result; and (iv) a far-field region away from the crack.

\section{Conclusions}

For beam-like geometries, the energy-release rate associated with axial loads and bending moments is unaffected by the nature of the bonding at the interface or by the properties of any interphase. The energy-release rate associated with transverse shear loading is affected by the crack-tip stress field and, hence, by the nature of the interfacial bonding. For most practical beam-like geometries, the effect of transverse shear at the crack tip is relatively small, and fracture is dominated by moments and axial loads. Provided the interface has mode-independent failure parameters, fracture for these geometries is governed only by toughness, and LEFM analyses provide excellent predictions for the strength even at very large fracture-length scales.

If an interface has mode-dependent properties, then LEFM invokes the notion of a nominal phase angle to describe mixed-mode fracture. This nominal phase angle, obtained by the use of stress-intensity factors, provides an excellent description of the partition of the energy-release rate into normal and shear components at the crack tip for fracture-length scales that encompass the range that would generally be expected in practical situations. The partition of the energy-release rate into normal and shear components using the nominal phase angle is valid, even when there is no inverse square-root singular stress field anywhere along the interface. The generality of this partition has the important consequence of indicating that failure loads of elastic beam-like geometries can be predicted accurately using the concepts of mixed-mode LEFM and a phase-angle-dependent toughness. If an interface is weak in shear, the mode-mixedness at the crack tip tends to a state of pure mode-I, so that the fracture behavior appears to be mode-independent, even when there is a difference between $\Gamma_{I}$ and $\Gamma_{I I}$.

There are loading conditions for which the crack tip in a beam-like geometry is predicted to be under a compressive normal stress, when the nominal phase angle has a magnitude of greater than $90^{\circ}$. Conventionally, in LEFM analyses, these conditions are dismissed as being physically unreal, because they would be associated with interpenetration of the crack surfaces. Instead, contact of the crack surfaces is assumed, with fracture occurring under pure mode-II conditions. The use of a cohesive 
zone in fracture introduces the physical reality that interfaces and interphases have a finite equilibrium thickness. When the cohesive zone is relatively thin, crack-surface contact prevents compression ahead of the crack tip, and fracture does occur under pure mode-II conditions. Consistent with existing LEFM solutions in the literature, the nominal toughness can be elevated slightly above the pure mode-II toughness because of frictional dissipation along the crack surfaces. Thicker cohesive layers can accommodate compression without crack-surface contact. If it is assumed that this compression does not contribute to fracture, but is lost when the mode-II failure condition is met, then the toughness can increase significantly above the mode-II value, without frictional effects occurring. This increase in toughness could be described by an LEFM failure criterion predicated on a nominal phase angle with a magnitude greater than $90^{\circ}$.

The discussions of the preceding paragraphs are valid when there is a modulus mismatch, such that $\alpha \neq 0$. However, there are additional complications associated with non-zero values of the other modulus mismatch parameter, $\beta$, which introduces oscillations in the stress and displacement fields. It is well-recognized in the literature that the predictions of crack-surface interpenetration must lead to crack-surface contact in an LEFM analysis. However, as described in the previous paragraph, the finite thickness associated with a cohesive-zone analysis allows for the possibility of fracture occurring without contact of the crack surfaces, and accommodation of compressive stresses along the interface. When $\beta \neq 0$, an additional length scale, that does not exist in LEFM analyses, is required to partition the energy-release rate into normal and shear components at the crack tip so that mixed-mode fracture can be analyzed. Cohesive-zone analyses introduce a cohesive strength into the description of the interface, and this naturally provides a length scale that allows an appropriate phase angle to exist at the crack tip. The length scale provides a relationship between the nominal phase angle (defined by LEFM in terms of a geometrical length) and the crack-tip phase angle, and is dependent on the values of the cohesive parameters. As this length scale is reduced, the shift between the nominal phase angle and the crack-tip phase angle increases. This shift can be relatively large and, therefore, $\beta$ can have a significant influence on the strength of interfaces.

\section{Acknowledgments}

MDT dedicates this paper to the memory of R. M. Cannon Jr. (1943-2006) in thanks for many delightful discussions about the physics of interfacial fracture. 


\section{References}

[1] C E Inglis. Stresses in a plate due to the presence of cracks and sharp corners. In Proceedings of the Institute of Naval Architects, volume 55, pages 219-230, 1913.

[2] J Cook and J E Gordon. A mechanism for the control of crack propagation in all-brittle systems. Proceedings of the Royal Society of London, Series A, 282:508-520, 1964.

[3] A A Griffith. The phenomenon of rupture and flow in solids. Philosophical Transactions of the Royal Society of London, A221:163-198, 1920.

[4] K. Kendall. The adhesion and surface energy of solids. Journal of Physics D, 4:1186-1195, 1971.

[5] J N Israelachvili. Direct measurement of a tethered ligand-receptor interaction potential. Science, 275:820-822, 1997.

[6] M S Kafkalidis, M D Thouless, Q D Yang, and S. M. Ward. Deformation and fracture of an adhesive layer constrained by plastically-deforming adherends. Journal of Adhesion Science and Technology, 14:1593-1607, 2000.

[7] M N Cavalli and M D Thouless. The effect of damage nucleation on the toughness of an adhesive joint. Journal of Adhesion, 76:75-92, 2001.

[8] C H Mastrangelo and C H Hsu. Mechanical stability and adhesion of microstructures under capillary forces - Part I: Basic theory. Journal of Microelectromechancial Systems, 2:33-43, 1993.

[9] S J Burns and B R Lawn. A simulated crack experiment illustrating the energy balance criterion. International Journal of Fracture Mechanics, 4:339-345, 1968.

[10] M D Thouless. Mixed-mode fracture of a lubricated interface. Acta Metallurgica et Materialia, 40:1281-1286, 1992.

[11] J W Hutchinson and Z Suo. Mixed mode cracking in layered materials. Advances in Applied Mechanics, 29:63-191, 1992.

[12] Q D Yang, M D Thouless, and S M Ward. Numerical simulations of adhesivelybonded beams failing with extensive plastic deformation. Journal of the Mechanics and Physics of Solids, 47:1337-1353, 1999.

[13] Q D Yang and M D Thouless. Mixed-mode fracture analyses of plasticallydeforming adhesive joints. International Journal of Fracture, 110:175-187, 2001. 
[14] Z Suo and J W Hutchinson. Interface crack between two elastic layers. International Journal of Fracture, 43:1-18, 1990.

[15] S Li, J Wang, and M D Thouless. The effects of shear on delamination in layered materials. Journal of the Mechanics and Physics of Solids, 52(1):193-214, 2004.

[16] G R Irwin. Analysis of stresses and strains near the end of a crack traversing a plate. Journal of Applied Mechanics, 24:361-364, 1957.

[17] V Tvergaard and J W Hutchinson. Toughness of ductile adhesive joints. Journal of the Mechanics and Physics of Solids, 44(5):789-800, 1996.

[18] A G Evans and J W Hutchinson. Effects of non-planarity on the mixed-mode fracture resistance of bimaterial interfaces. Acta Metallurgica et Materialia, 37:909916, 1989.

[19] K T Faber and A G Evans. Crack deflection processes - I. theory. Acta Metallurgica, 31(4):565-576, 1983.

[20] M D Thouless. Fracture of a model interface under mixed-mode loading. Acta Metallurgica et Materialia, 38:1135-1140, 1990.

[21] J. S. Wang and Z. Suo. Experimental determination of interfacial toughness using brazil-nut-sandwich. Acta Metallurgica et Materialia, 38:1279-1290, 1990.

[22] K M Liechti and Y S Chai. Asymmetric shielding in interfacial fracture under in-plane shear. Journal of Applied Mechanics, 59:295-304, 1992.

[23] J Dundurs. Edge-bonded dissimilar orthogonal elastic wedges. Journal of Applied Mechanics, 36:650-652, 1969.

[24] J R Rice and G C Sih. Plane problems of cracks in dissimilar media. Journal of Applied Mechanics, 32:418-423, 1965.

[25] J R Rice. Elastic fracture-mechanics concepts for interfacial cracks. Journal of Applied Mechanics, 55(1):98-103, 1988.

[26] Z Suo and J W Hutchinson. On sandwich test specimen for measuring interface crack toughness. Materials Science and Engineering, A107:135-143, 1989.

[27] N A Fleck, J W Hutchinson, and Z Suo. Crack path selection in a brittle adhesive layer. International Journal of Solids and Structures, 27:455-466, 1991.

[28] J W Hutchinson, M E Mear, and J R Rice. Crack paralleling an interface between dissimilar materials. Journal of Applied Mechanics, 54:828-832, 1987. 
[29] B Cotterell and J R Rice. Slightly curved or kinked cracks. International Journal of Fracture, 16(2):155-169, 1980.

[30] M D Thouless, J W Hutchinson, and E G Liniger. Plane strain buckling-driven delamination of thin films: Model experiments and mode-II fracture. Acta Metallurgica et Materialia, 40:2639-2649, 1992.

[31] M D Thouless, H M Jensen, and E G Liniger. Delamination from edge flaws. Proceedings of the Royal Society, A447:271-279, 1994.

[32] G Fernlund and J K Spelt. Mixed-mode fracture characterization of adhesive joints. Composites Science and Technology, 50(4):441-449, 1994.

[33] A Needleman. A continuum model for void nucleation by inclusion debonding. Journal of Applied Mechanics, 54:525-553, 1987.

[34] V Tvergaard and J W Hutchinson. The relation between crack growth resistance and fracture process parameters in elastic plastic solids. Journal of the Mechanics and Physics of Solids, 40:1377-1392, 1992.

[35] D S Dugdale. Yielding of sheets containing slits. Journal of the Mechanics and Physics of Solids, 8:100-104, 1960.

[36] G I Barenblatt. The mathematical theory of equilirium cracks in brittle fracture. Advances in Applied Mechanics, 7:55-129, 1962.

[37] Z-H Jin and C T Sun. Cohesive zone modeling of interface fracture in elastic bi-materials. Engineering Fracture Mechanics, 72:1805-1817, 2005.

[38] T Andersson and U Stigh. The stress-elongation relation for an adhesive layer loaded in peel using equilibrium of energetic forces. International Journal of Solids and Structures, 41(2):413-434, 2004.

[39] J. Parmigiani. Delamination and Deflection at Interfaces. PhD thesis, University of Michigan, Ann Arbor, MI, 2007.

[40] M D Thouless. Bridging and damage zones in crack growth. Journal of the American Ceramic Society, 71:408-413, 1988.

[41] H Tada, P C Paris, and G R Irwin. The Stress Analysis of Cracks Handbook, 2nd ed. Paris Productions, Inc., St. Louis, MO, 1985.

[42] G Bao and Z Suo. Remarks on crack-bridging concepts. Applied Mechanics Review, 45:355-366, 1992. 
[43] T L Becker Jr., J. M. McNaney, R. M. Cannon, and R. O. Ritchie. Limitations on the use of the mixed-mode delaminating beam test specimen: Effects of the size of the region of K-dominance. Mechanics of Materials, 25:291-308, 1997.

[44] J W Hutchinson, M D Thouless, and E G Liniger. Growth and configurational stability of circular, buckling-driven film delaminations. Acta Metallurgica et Materialia, 40:295-308, 1992.

[45] H M Jensen and M D Thouless. Effects of residual stresses in the blister test. International Journal of Solids and Structures, 30:779-795, 1993.

[46] R G Stringfellow and L B Freund. The effect of interfacial friction on the buckledriven spontaneous delamination of a compressed thin film. International Journal of Solids and Stuctures, 30:1379-1395, 1993.

[47] M Comninou. An overview of interface cracks. Engineering Fracture Mechanics, 37(1):197-208, 1990.

[48] B M Malyshev and R L Salganik. The strength of adhesive joints using the theory of fracture. International Journal of Fracture Mechanics, 1:114-128, 1965. 


\section{Figure Captions}

Figure 1: (a) A layered material loaded by an axial load and moment. (b) A layered material loaded by a transverse shear load.

Figure 2: Schematic illustration of the (a) mode-I, and (b) mode-II tractionseparation laws used for the cohesive-zone model in this paper. Throughout this paper the values of $\delta_{n_{1}} / \delta_{n_{c}}=\delta_{t_{1}} / \delta_{t_{c}}$ and $\delta_{n_{2}} / \delta_{n_{c}}=\delta_{t_{2}} / \delta_{t_{c}}$ were kept at fixed values of 0.01 and 0.75 respectively.

Figure 3: (a) Plot of normalized strength as a function of normalized fracturelength scale, $\bar{E} \Gamma_{I} / \hat{\sigma}^{2} a$, for a mode-I geometry with a central crack of length $2 a$, a ligament length $L$, and subjected to a tensile load $P$. The non-dimensional parameters are $\Gamma_{I} / \bar{E} a=9.1 \times 10^{-7}, a / L=0.9$, $H / L=20$ and $H_{o} / L=7.4$. One set of data shows the strength normalized by the fracture load predicted by LEFM, $P_{L E F M}$. The other set shows the strength normalized by the fracture load predicted from the cohesive strength of the interface, $P_{\text {strength }}$. For the purposes of comparison, $P_{L E F M} / P_{\text {strength }}$ and $P_{\text {strength }} / P_{L E F M}$ are superimposed on this figure to illustrate the asymptotic limits for the strength based on the two models of fracture. (b) Plots of the normal traction distribution along the interface of the mode-I central cracked geometry, for two extreme values of the fracture-length scale, $\bar{E} \Gamma_{I} / \hat{\sigma}^{2} a$.

Figure 4: (a) Fracture load, $P_{f}$, normalized by the strength predicted by LEFM, $P_{L E F M}$, for a mixed-mode geometry consisting of a tensile plate with a central crack of length $2 a$, ligaments of length $L$, and subjected to a tensile load $P$ and a shear load $Q$. (b) The crack-tip phase angle at fracture for this mixed-mode geometry, plotted as a function of fracture-length scale. The non-dimensional groups for these two figures are $\Gamma_{I} / \bar{E} a=9.1 \times 10^{-7}, \Gamma_{I I} / \Gamma_{I}=1, \hat{\sigma} / \hat{\tau}=1, Q / P=1, a / L=0.9$, $H / L=20$ and $H_{o} / L=7.4$.

Figure 5: Plots of the (a) normal and (b) shear traction distribution along the interface of the mixed-mode central-cracked geometry, for different values of the fracture-length scale, $\bar{E} \Gamma_{I} / \hat{\sigma}^{2} a$.

Figure 6: (a) The fracture load for an asymmetrical double-cantilever beam plotted as a function of ligament length for three different values of the fracture-length scale, $\bar{E} \Gamma_{I} / \hat{\sigma}^{2} h_{1}$, and with other non-dimensional parameters given by $\Gamma_{I} / \bar{E} h_{1}=9.1 \times 10^{-7}, \Gamma_{I I} / \Gamma_{I}=1.0, \hat{\tau} / \hat{\sigma}=1.0$, 
$h_{1} / h_{2}=0.1$, and $a / h_{1}=20$. (b) A plot of the fracture load for an asymmetrical double-cantilever beam plotted as a function of the fracture-length scale for $\Gamma_{I} / \bar{E} h_{1}=9.1 \times 10^{-7}, h_{1} / h_{2}=0.1, \Gamma_{I I} / \Gamma_{I}=$ 1.0, $\hat{\tau} / \hat{\sigma}=1.0, a / h_{1}=400$, and $L / h_{1}=130$. The results presented in both figures are compared to the LEFM results for an asymmetrical double-cantilever beam with $h_{1} / h_{2}=0.1$.

Figure 7: The failure load plotted as a function of fracture-length scale and different values of the ratio of the mode-II to mode-I toughness for an asymmetrical-double-cantilever beam loaded by (a) a transverse shear load only and (b) a transverse shear load in conjunction with a fixed axial load of $P / \Gamma_{I}=833$. The LEFM predictions for the failure loads are indicated by dashed lines on both plots. The non-dimensional groups for these plots are $\Gamma_{I} / \bar{E} h_{1}=9.1 \times 10^{-7}, \hat{\tau} / \hat{\sigma}=1.0, h_{1} / h_{2}=0.1$, $a / h_{1}=20$, and $L / h_{1}=130$.

Figure 8: The crack-tip phase angle at fracture plotted as a function of fracturelength scale and different values of the ratio of the mode-II to mode-I toughness for an asymmetrical double-cantilever beam loaded by (a) a transverse shear load only and (b) a transverse shear load in conjunction with a fixed axial load of $P / \Gamma_{I}=833$. The LEFM predictions for the nominal phase angles are indicated by dashed lines on both plots. The non-dimensional groups for these plots are $\Gamma_{I} / \bar{E} h_{1}=9.1 \times 10^{-7}$, $\hat{\tau} / \hat{\sigma}=1.0, h_{1} / h_{2}=0.1, a / h_{1}=20$, and $L / h_{1}=130$.

Figure 9: Plots of how (a) the normal and shear tractions at fracture, and (b) the phase angle vary with distance along the interface of an asymmetricaldouble-cantilever with $\bar{E} \Gamma_{I} / \hat{\sigma}^{2} h_{1}=1.43, \Gamma_{I} / \bar{E} h_{1}=9.1 \times 10^{-7}, \Gamma_{I I} / \Gamma_{I}=$ 1.0, $\hat{\tau} / \hat{\sigma}=1.0, h_{1} / h_{2}=0.1, a / h_{1}=20$, and $L / h_{1}=130$. The tractions are compared with the expected LEFM singular field at fracture.

Figure 10: Plots of (a) the fracture load and (b) the crack-tip phase angle for an asymmetrical-double-cantilever beam plotted as a function of the ratio of the shear strength to the normal strength for different values of the ratio of the mode-II to mode-I toughness. The LEFM results for both figures are indicated by dashed lines. The non-dimensional groups for these plots are $\bar{E} \Gamma_{I} / \hat{\sigma}^{2} h_{1}=0.148, \Gamma_{I} / \bar{E} h_{1}=9.1 \times 10^{-7}, h_{1} / h_{2}=0.1$, $a / h_{1}=15$, and $L / h_{1}=135$.

Figure 11: (a) The results of a numerical experiment conducted on an asymmetric double-cantilever geometry, showing the values of axial and trans- 
verse shear loads required for fracture. (b) Plots of nominal toughness against nominal phase angle, with the LEFM mixed-mode failure criterion superimposed. (c) A comparison between the crack-tip phase angle at fracture and the nominal phase angle calculated from LEFM. The non-dimensional groups for these plots are $\bar{E} \Gamma_{I} / \hat{\sigma}^{2} h_{1}=0.055$, $\Gamma_{I} / \bar{E} h_{1}=9.1 \times 10^{-7}, \Gamma_{I I} / \Gamma_{I}=5, \hat{\tau} / \hat{\sigma}=0.5, h_{1} / h_{2}=0.1, a / h_{1}=20$, and $L / h_{1}=130$. Results for different values of the cohesive-zone thickness, $t$, and the coefficient of fraction $\mu$, are shown on all the plots.

Figure 12: The (a) normal and (b) shear stresses ahead of a crack tip at fracture for an asymmetric double-cantilever geometry subject to axial loads, with a modulus mismatch of $\alpha=0.8$ and $\beta=0.4,0.3,0.2,0.1$, and 0 . The corresponding LEFM stresses are superimposed on this figure. The non-dimensional groups for of these plots are $\bar{E} \Gamma_{I} / \hat{\sigma}^{2} h_{1}=0.1$, $\Gamma_{I} / \bar{E} h_{1}=10^{-6}, \Gamma_{I I} / \Gamma_{I}=1, \hat{\tau} / \hat{\sigma}=1, h_{1} / h_{2}=0.1, a / h_{1}=20$, and $L / h_{1}=130$.

Figure 13: The effects of modulus mismatch on the fracture load and the cracktip phase angle at fracture for an asymmetric double-cantilever geometry subjected to a couple formed by axial loads with (a) $\beta=0$, (b) $\beta=\alpha / 2$ (c) $\alpha=0.8$. The non-dimensional groups for these plots are $\bar{E} \Gamma_{I} / \hat{\sigma}^{2} h_{1}=0.1, \Gamma_{I} / \bar{E} h_{1}=10^{-6}, \Gamma_{I I} / \Gamma_{I}=1, \hat{\tau} / \hat{\sigma}=1, h_{1} / h_{2}=0.1$, $a / h_{1}=20$, and $L / h_{1}=130$.

Figure 14: The effects of modulus mismatch on the fracture load and the cracktip phase angle at fracture for an asymmetric double-cantilever geometry subjected to a couple formed by axial loads with $\alpha=0$ and various values of $\beta$. The non-dimensional groups for these plots are $\bar{E} \Gamma_{I} / \hat{\sigma}^{2} h_{1}=0.1, \Gamma_{I} / \bar{E} h_{1}=10^{-6}, \Gamma_{I I} / \Gamma_{I}=5, \hat{\tau} / \hat{\sigma}=1, h_{1} / h_{2}=0.1$, $a / h_{1}=20$, and $L / h_{1}=130$.

Figure 15: The shift in phase angle at fracture between the nominal value, $\psi^{\infty}$, defined by LEFM at a length scale of $h_{1}$, and the crack-tip phase angle, $\psi_{o}$, determined from the cohesive-zone model, plotted as a function of modulus-mismtach parameter $\epsilon=(1 / 2 \pi) \ln (1-\beta) /(1+\beta)$. All the data from Figs. 13 and 14 have been included on this figure.

Figure 16: The phase angle defined by Eqn. 8 plotted as a function of distance ahead of the crack for an asymmetric double-cantilever geometry subjected to a couple formed by axial loads. Two plots are shown: one for a modulus mismatch of $\alpha=0.4$ and $\beta=0.2$; the other for a modulus 
mismatch of $\alpha=0.4$ and $\beta=0.0$. The LEFM predictions for the phase angle are superimposed on this plot. The non-dimensional groups for these plots are $\bar{E} \Gamma_{I} / \hat{\sigma}^{2} h_{1}=0.1, \Gamma_{I} / \bar{E} h_{1}=10^{-6}, \Gamma_{I I} / \Gamma_{I}=1, \hat{\tau} / \hat{\sigma}=1$, $h_{1} / h_{2}=0.1, a / h_{1}=20$, and $L / h_{1}=130$. 


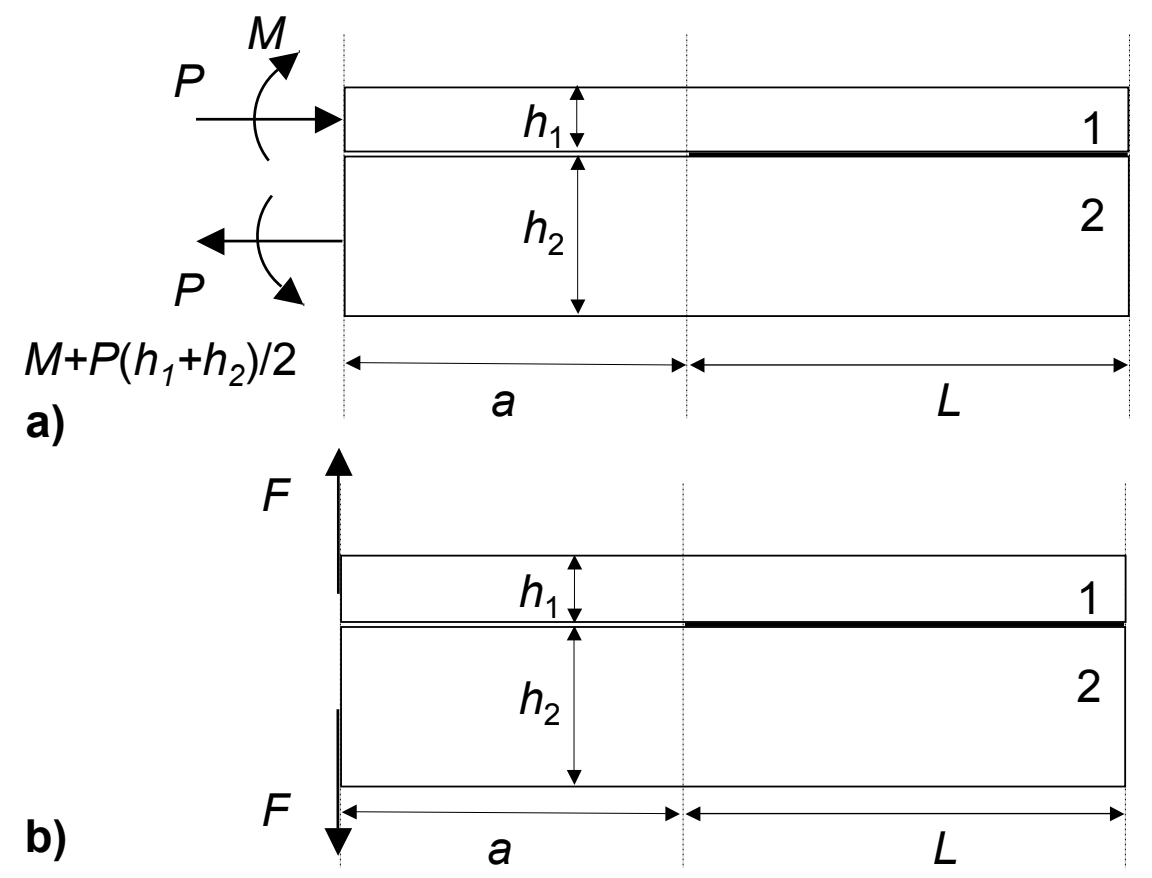

Figure 1: 
(a)

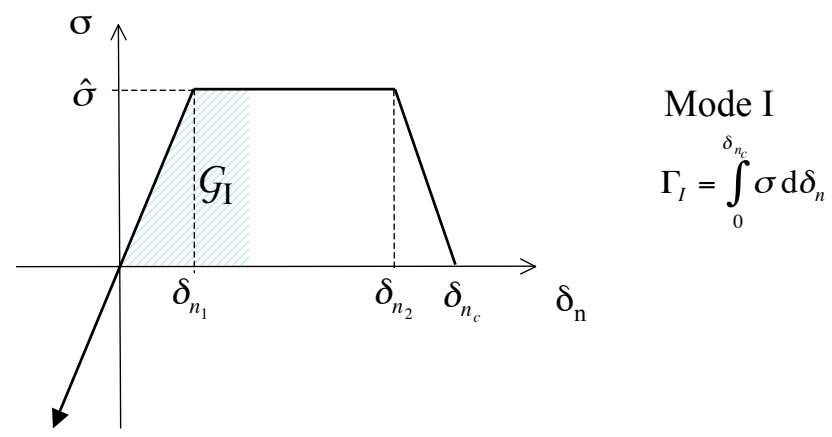

(b)

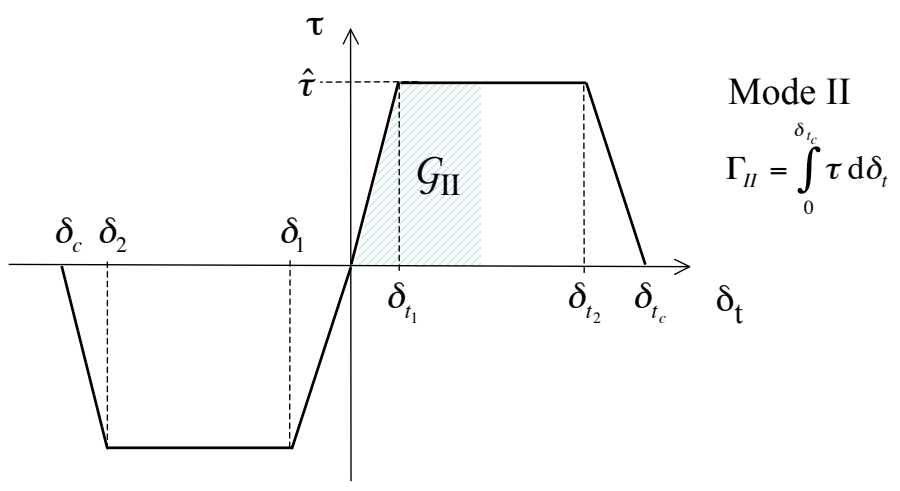

Figure 2: 


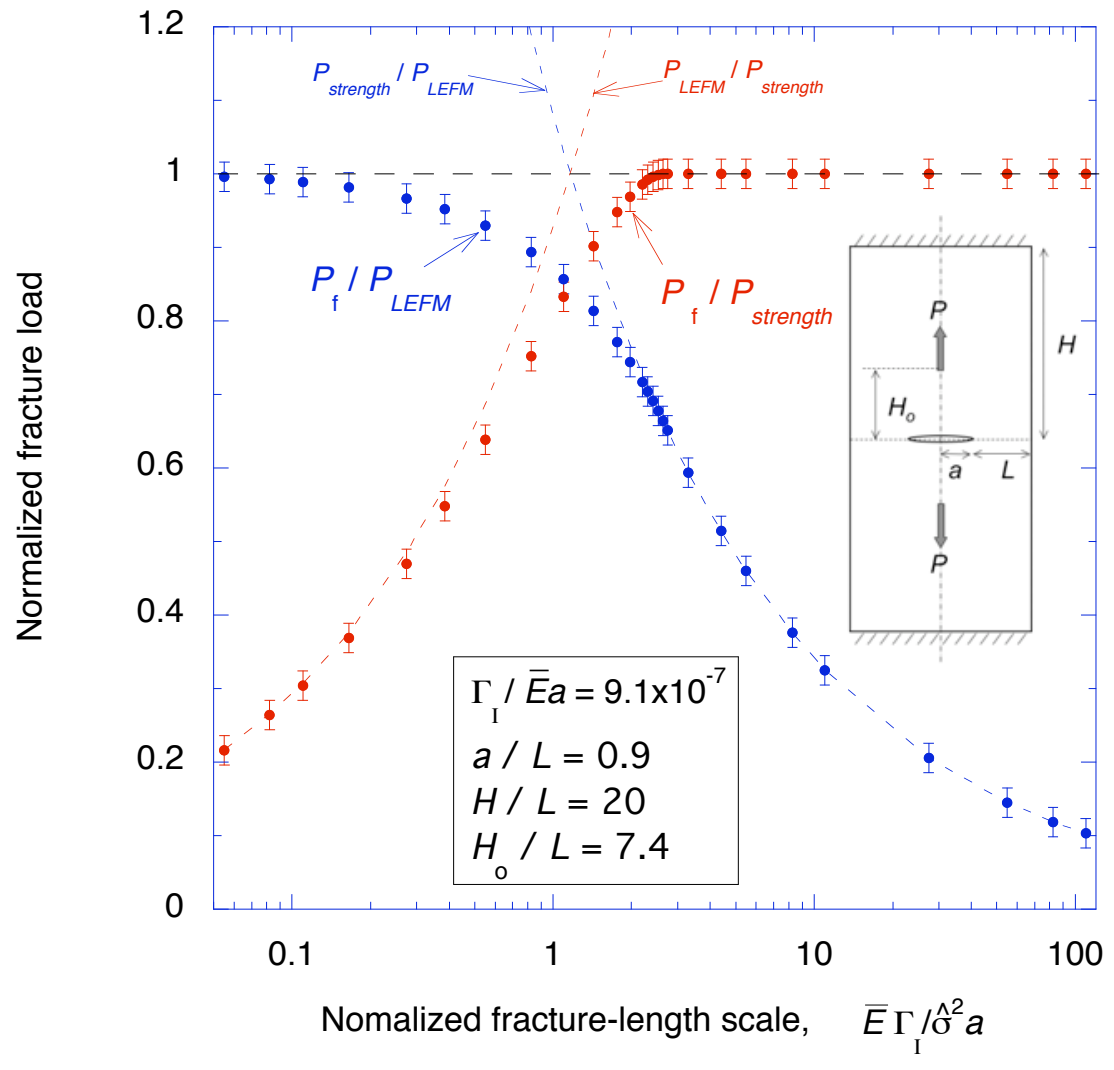

Figure 3: (a) 


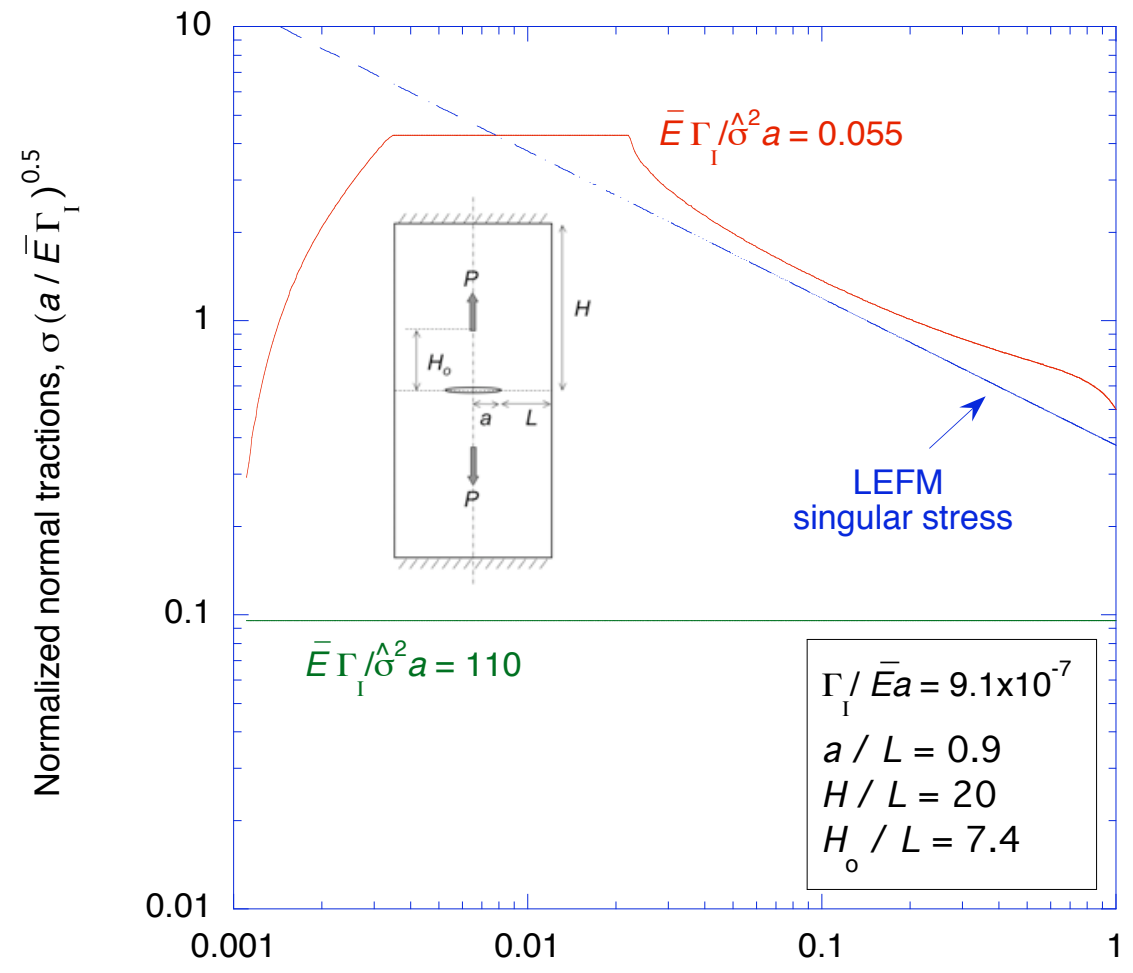

Normalized distance ahead of crack tip, $r / L$

Figure 3: (b) 


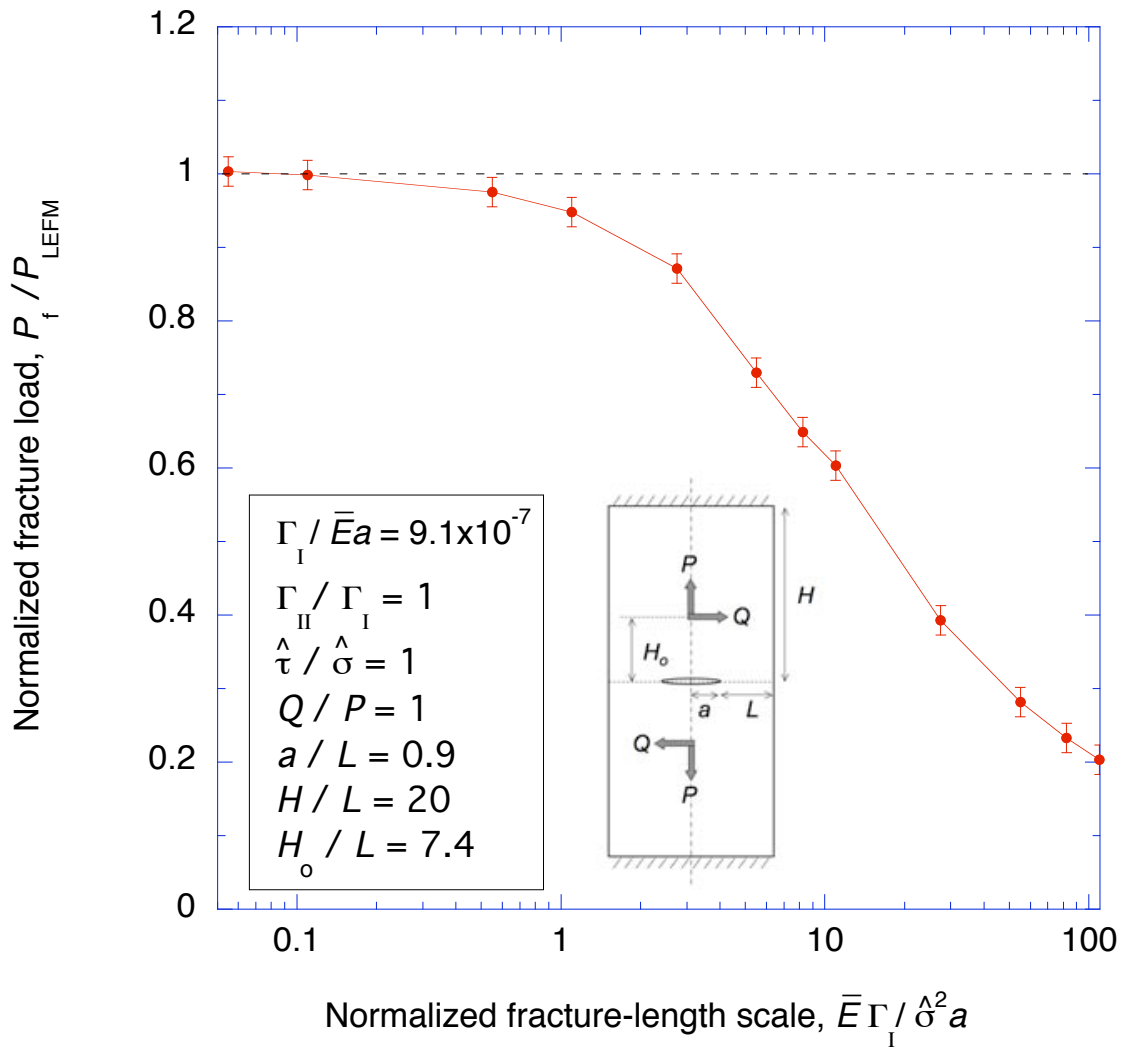

Figure 4: (a) 


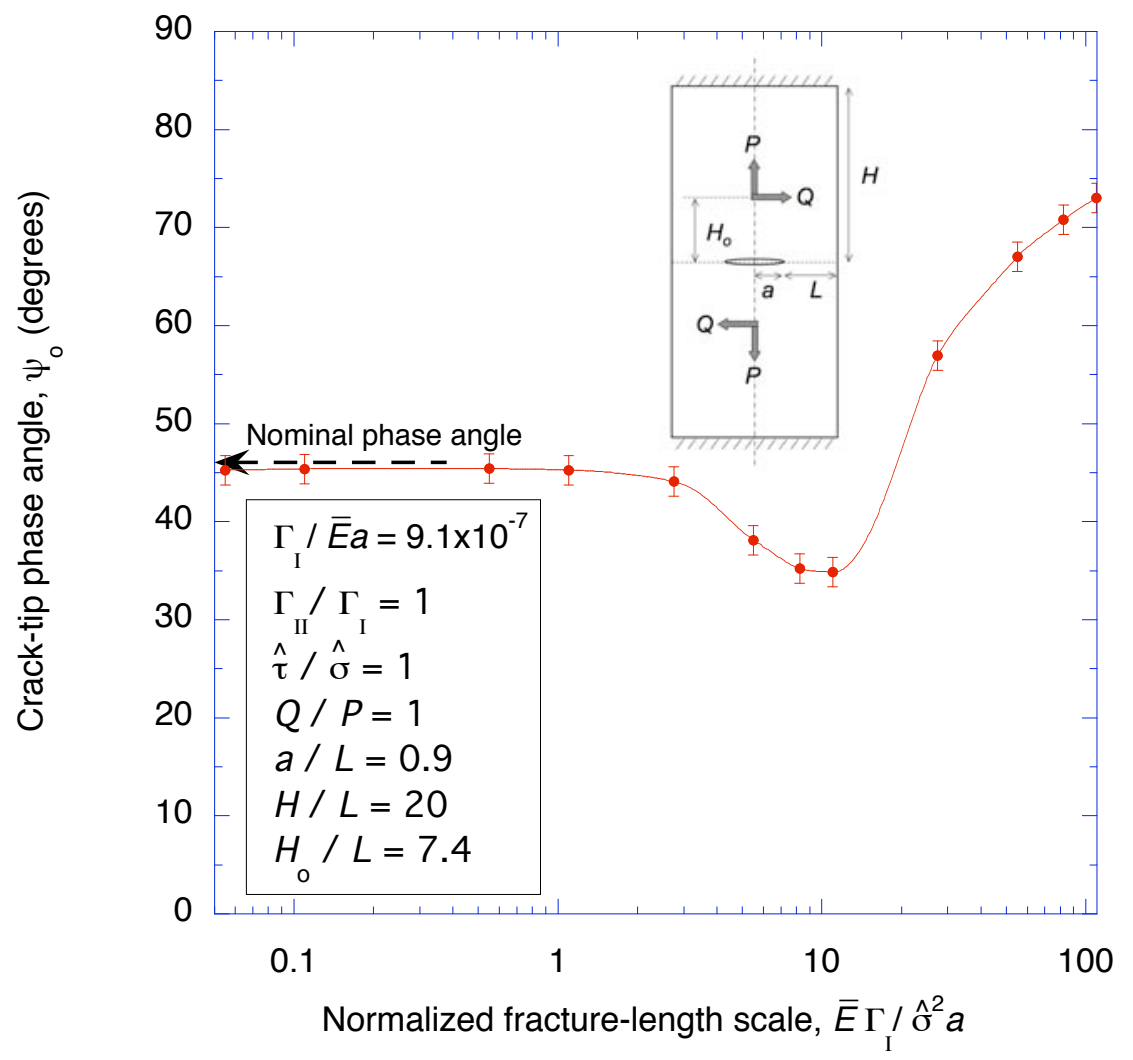

Figure 4: (b) 


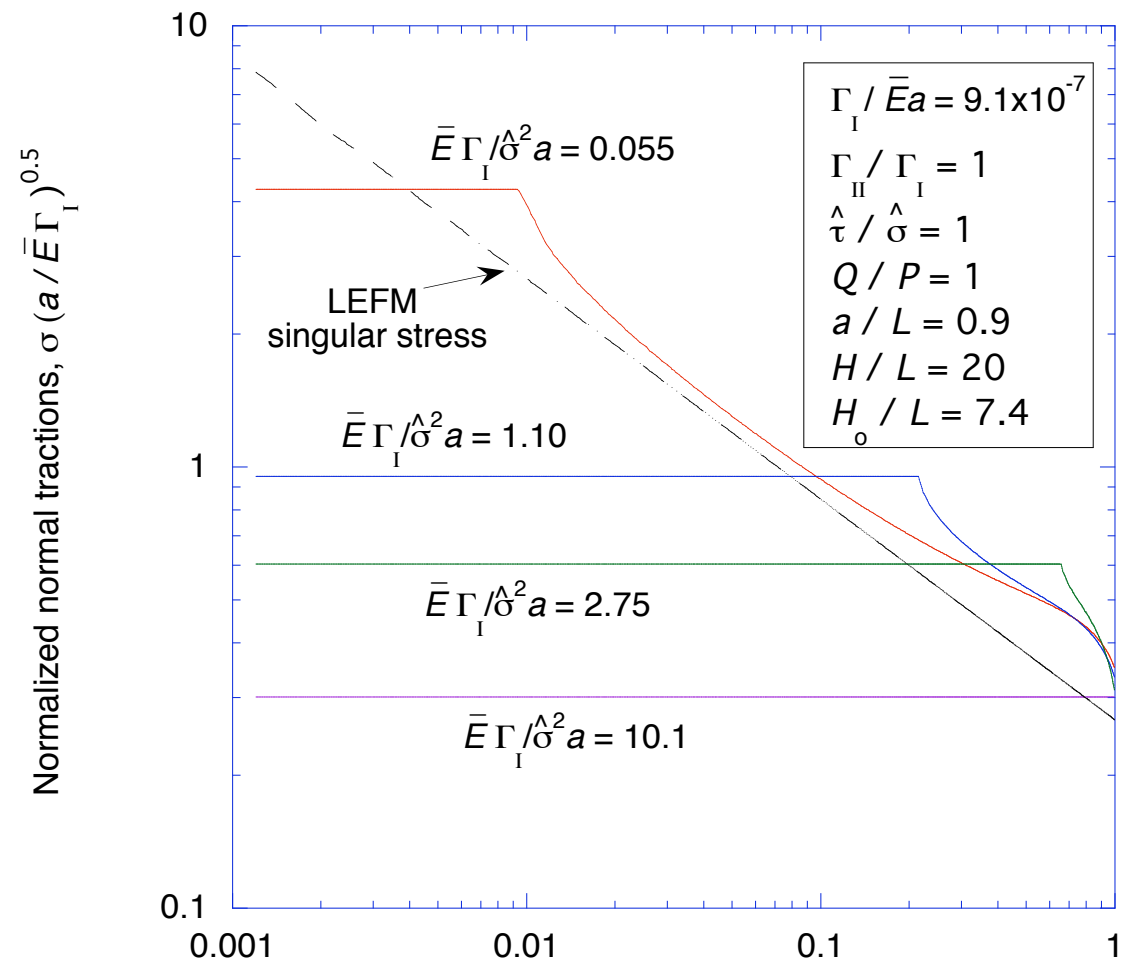

Normalized distance ahead of crack tip, $r$ / $L$

Figure: 5(a) 


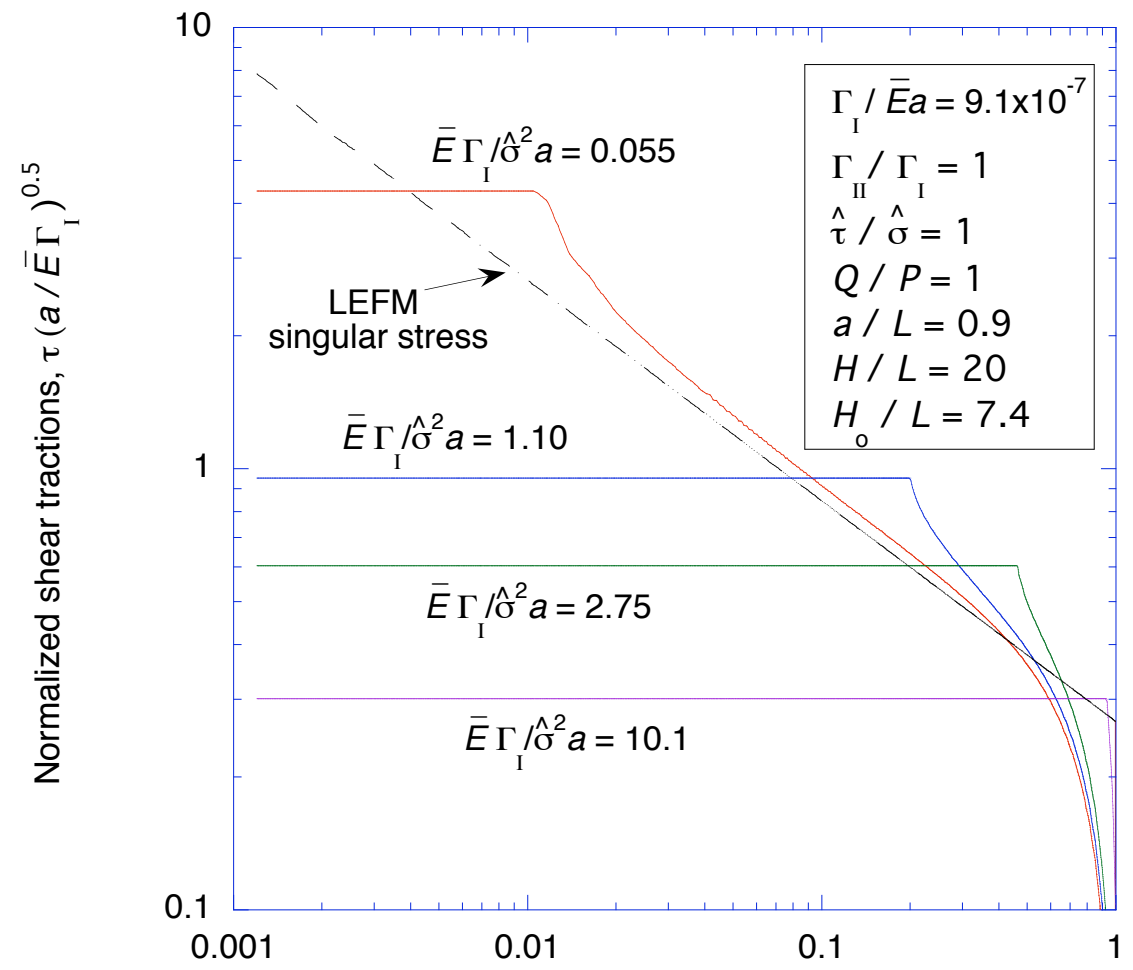

Normalized distance ahead of crack tip, $r / L$

Figure 5: (b) 


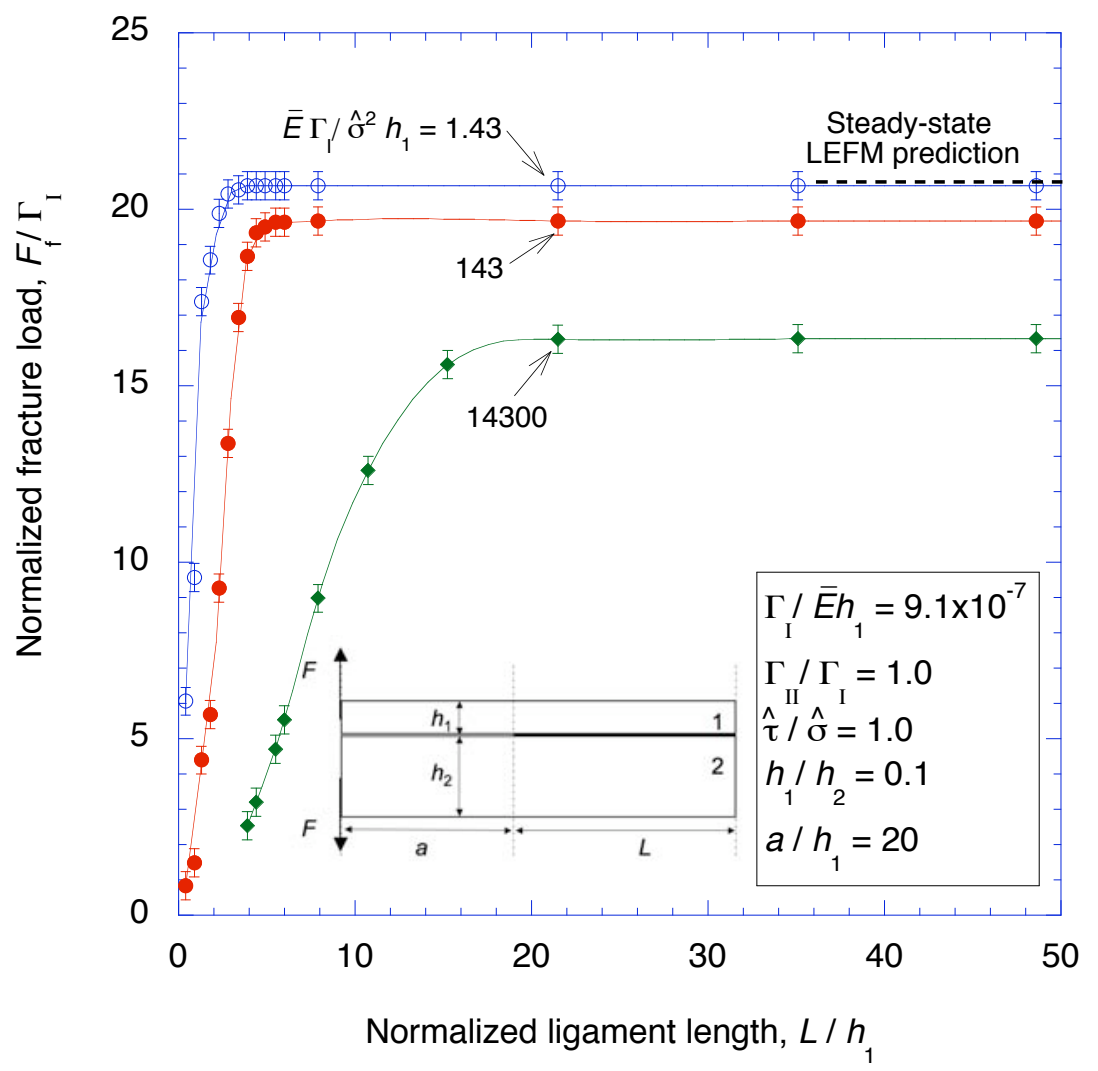

Figure 6: (a) 


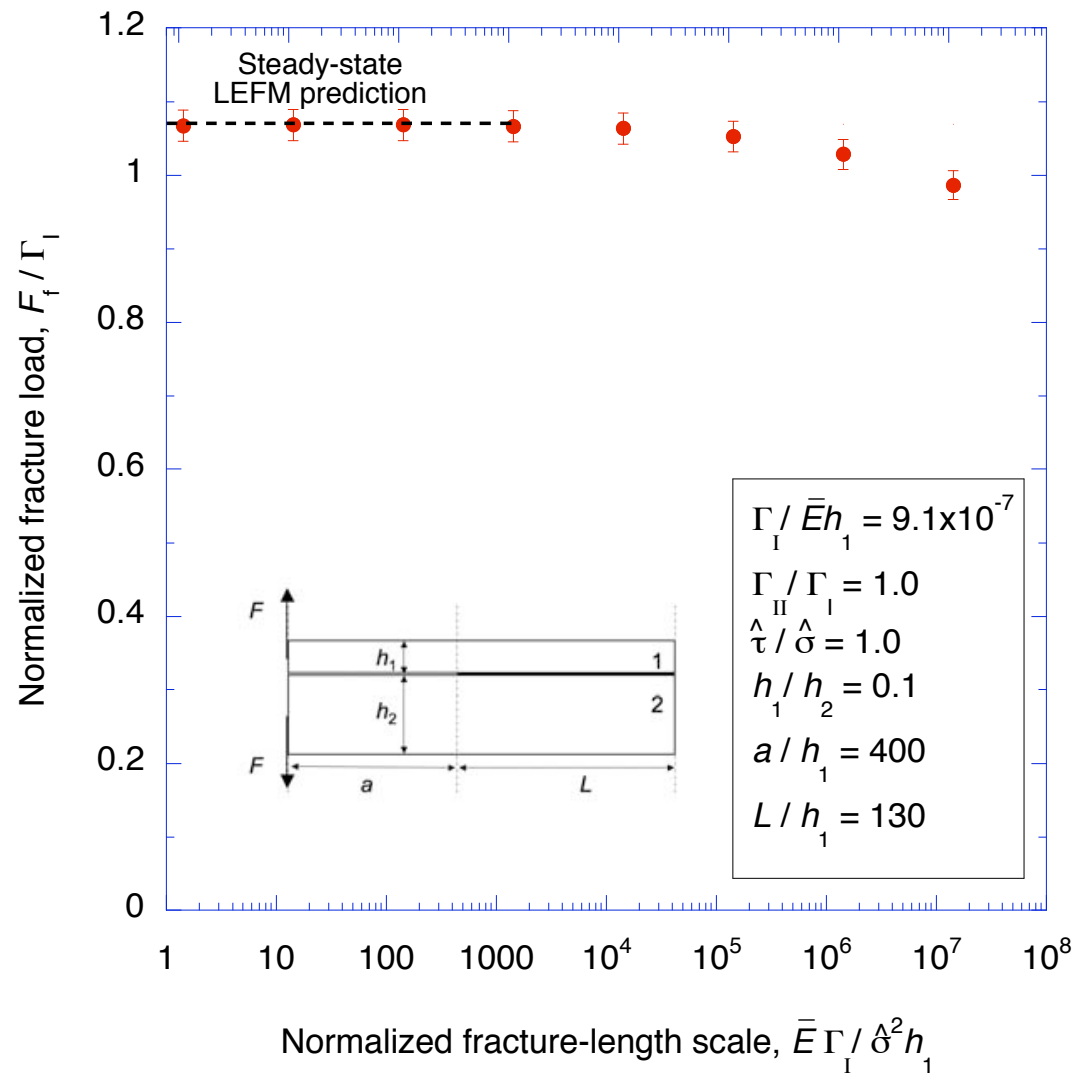

Figure 6: (b) 


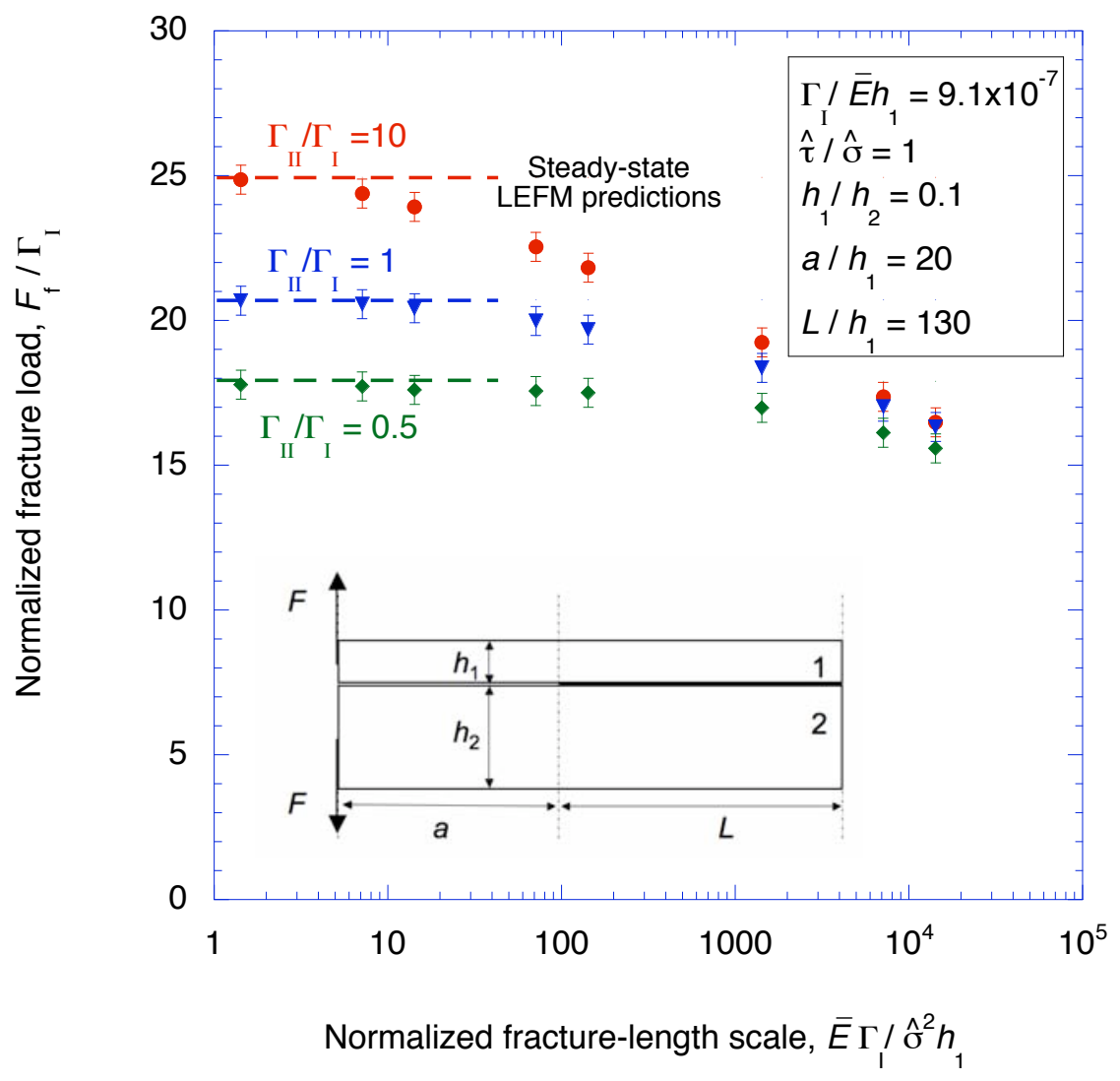

Figure 7: (a) 


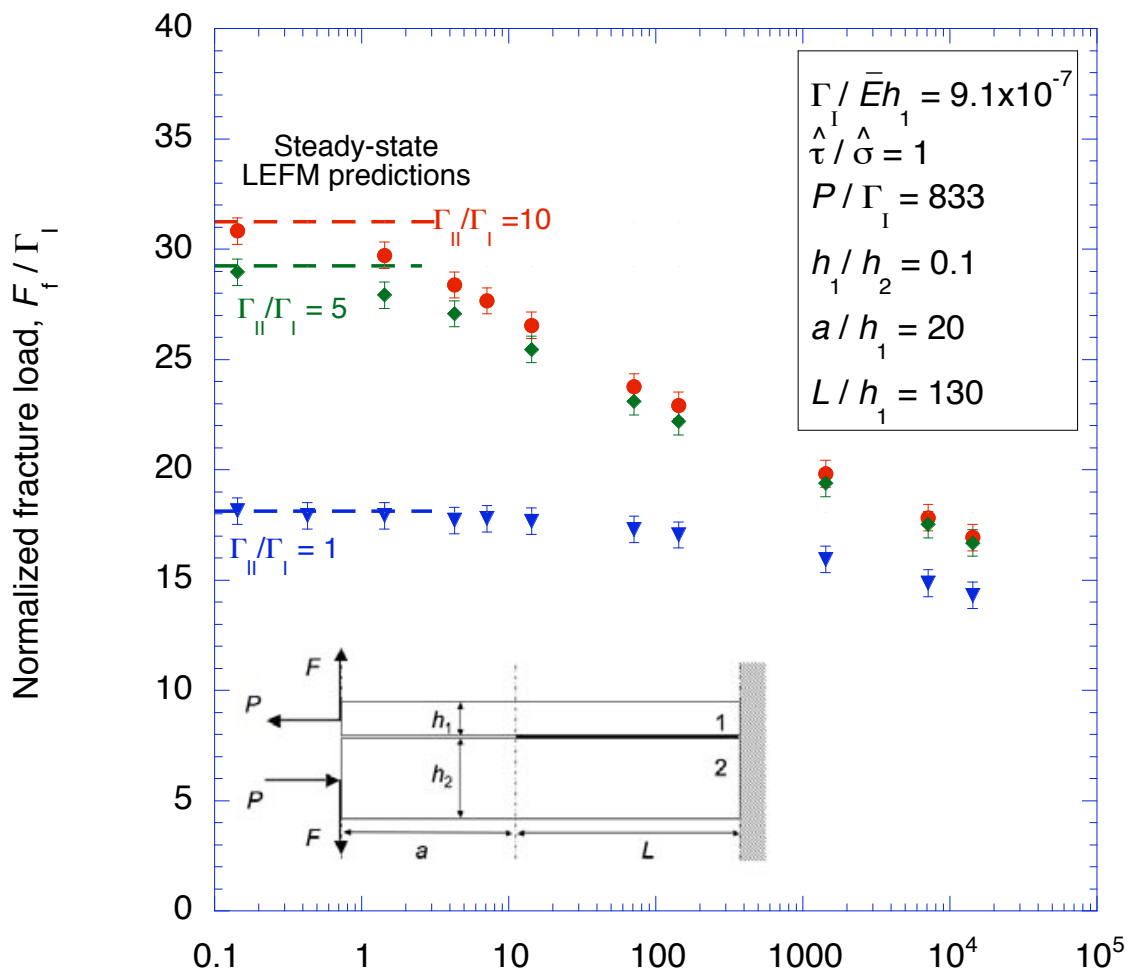

Normalized fracture-length scale, $\bar{E} \Gamma_{I}{\stackrel{\wedge}{\sigma} h_{1}}^{2}$

Figure 7: (b) 


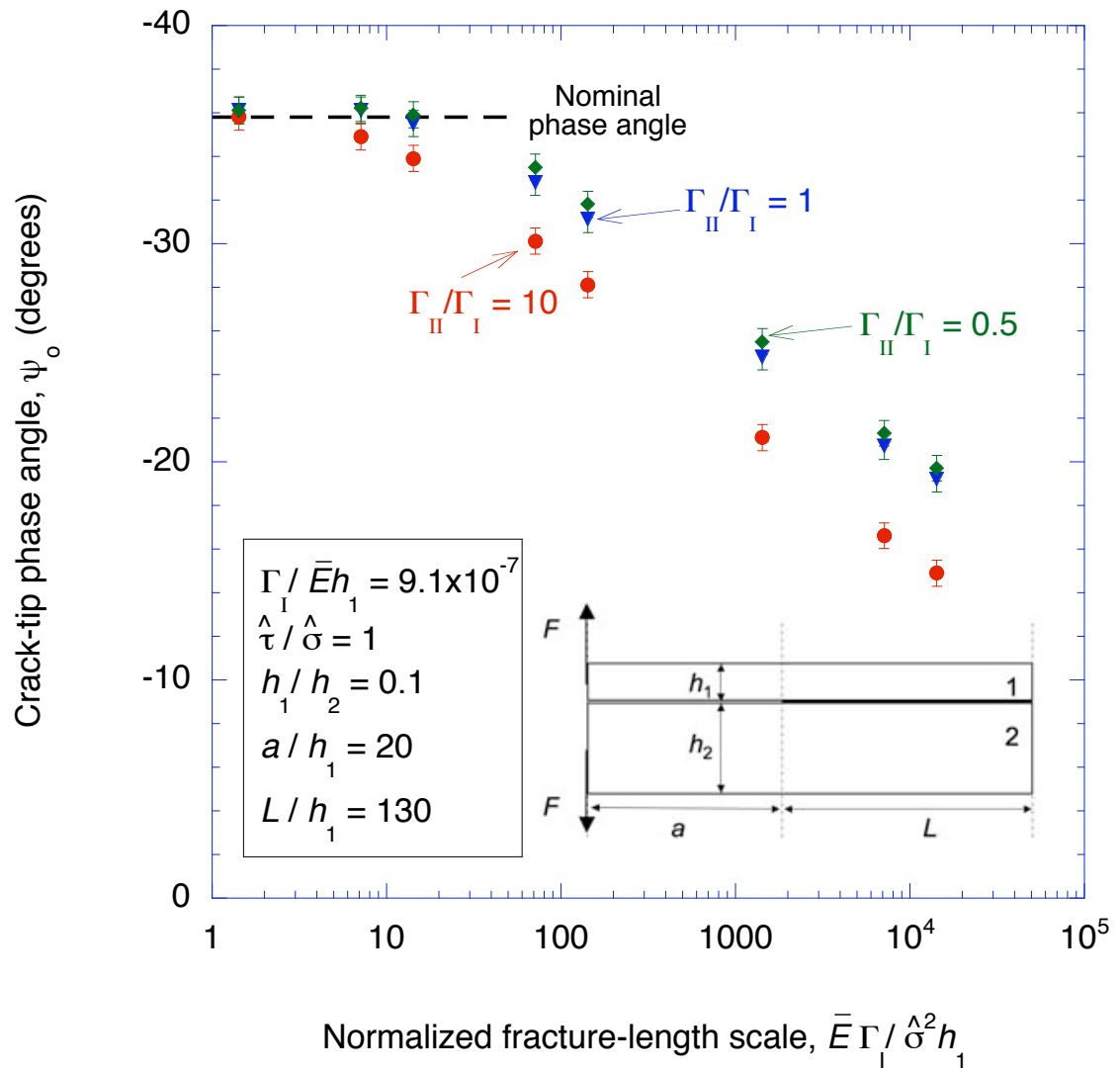

Figure 8: (a) 


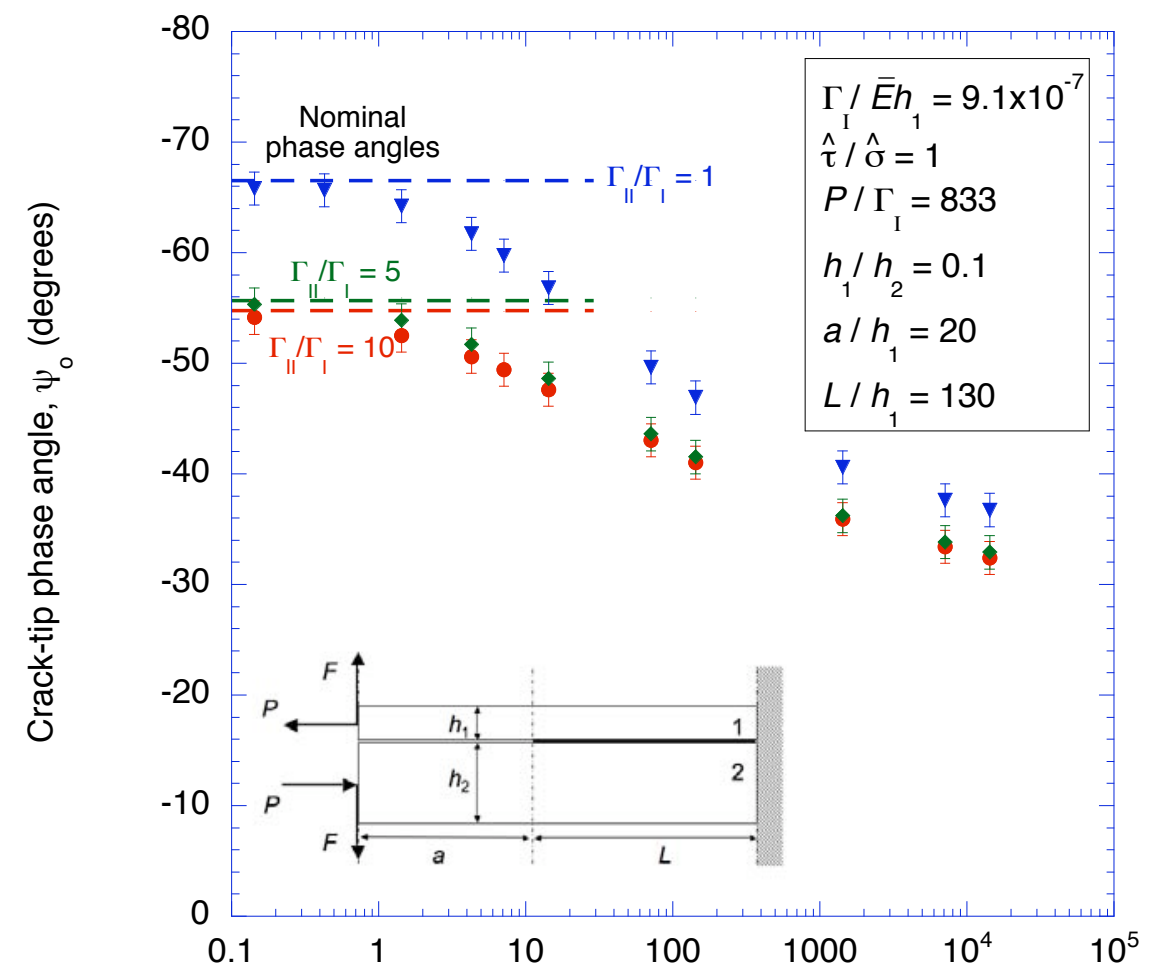

Normalized fracture-length scale, $\bar{E} \Gamma_{1} / \hat{\sigma}^{2} h_{1}$

Figure 8: (b) 


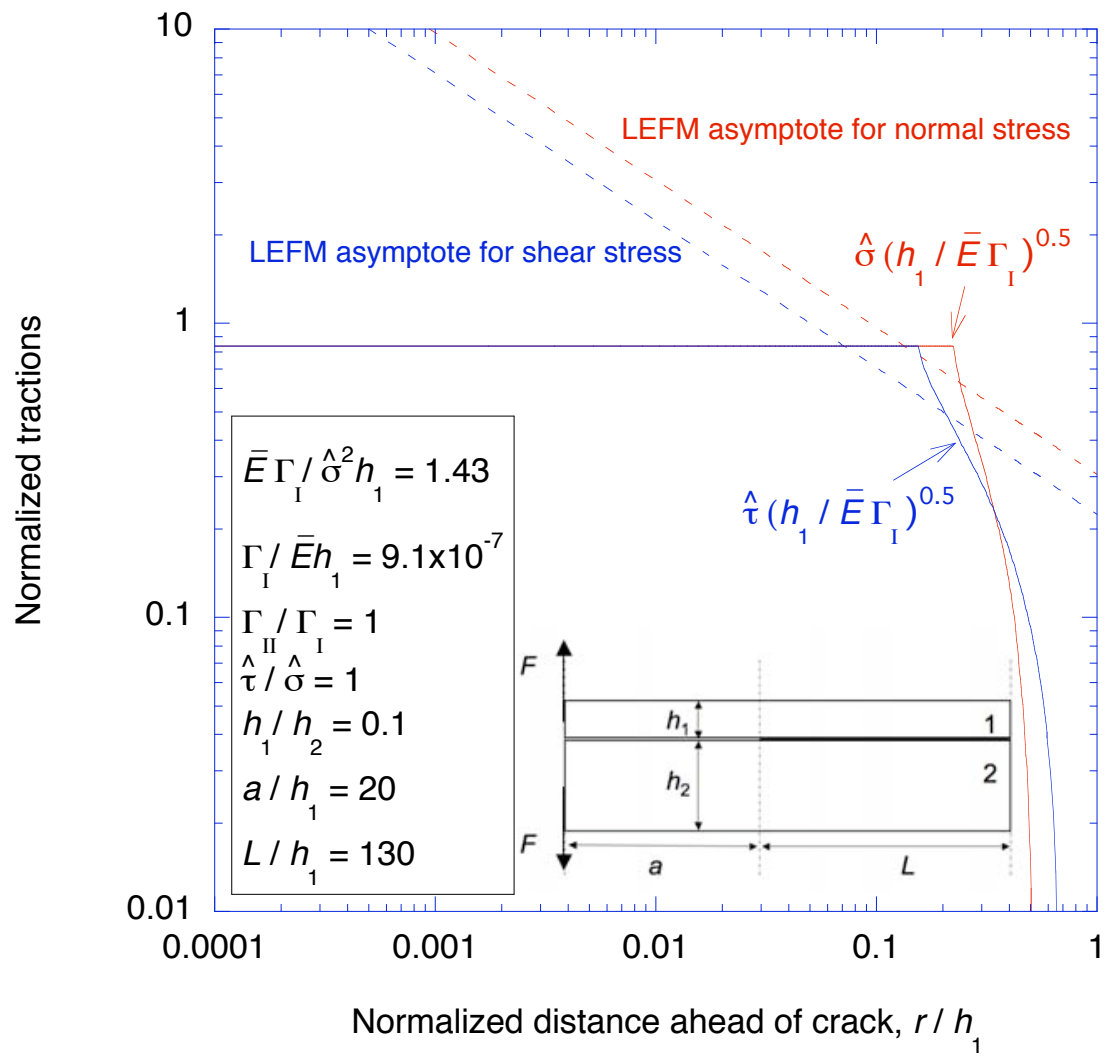

Figure 9: (a) 


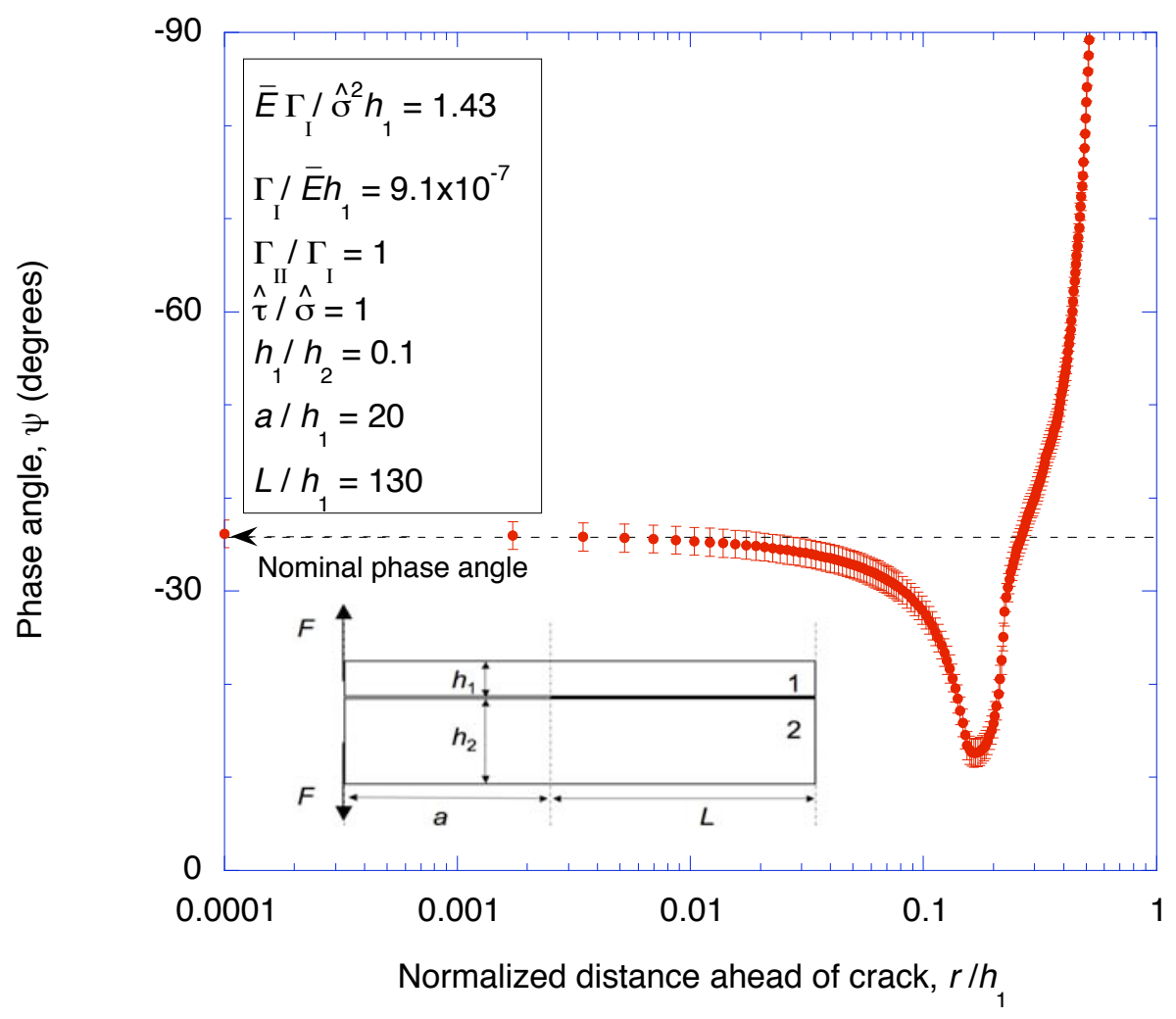

Figure 9: (b) 


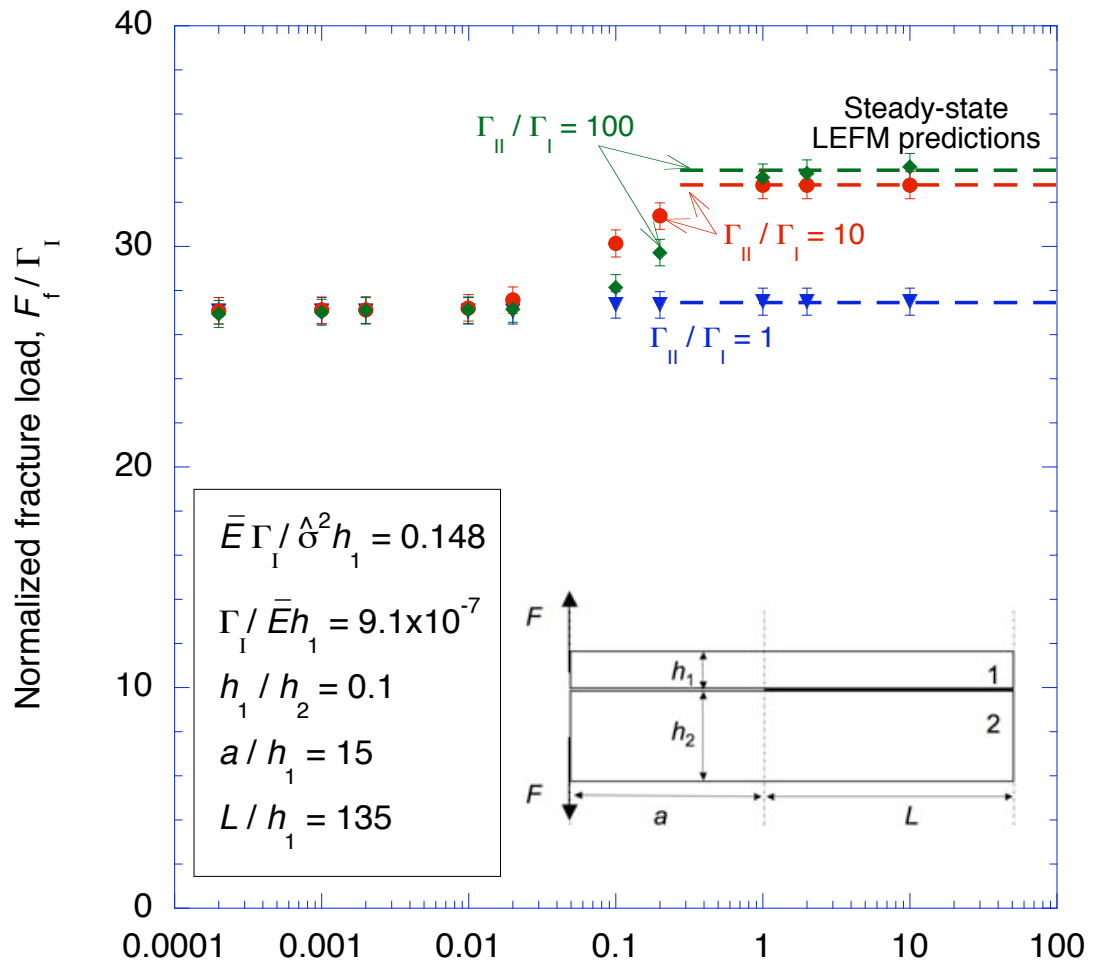

Ratio of shear to normal cohesive strength, $\hat{\tau} / \hat{\sigma}$

Figure 10: (a) 


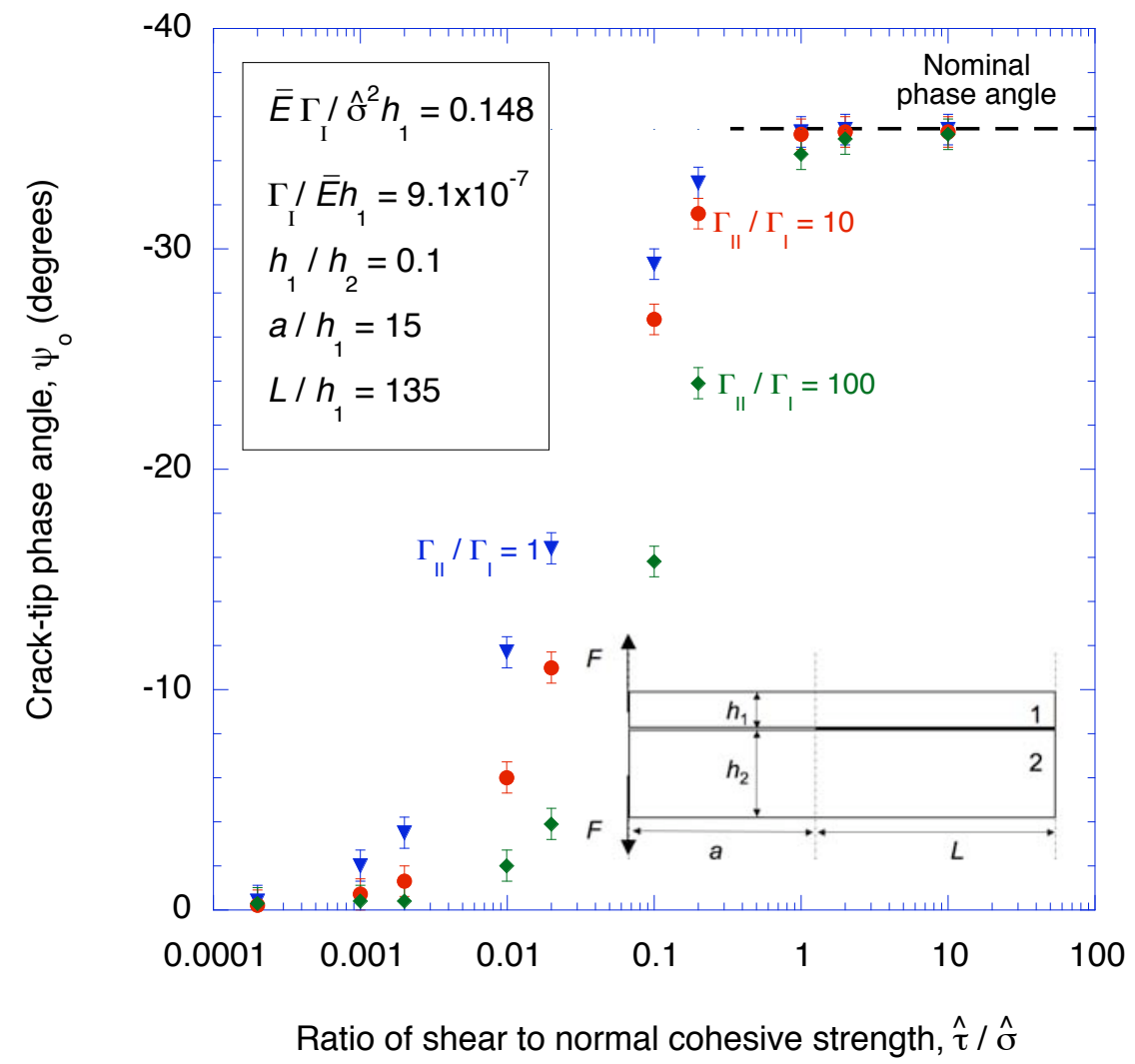

Figure 10: (b) 


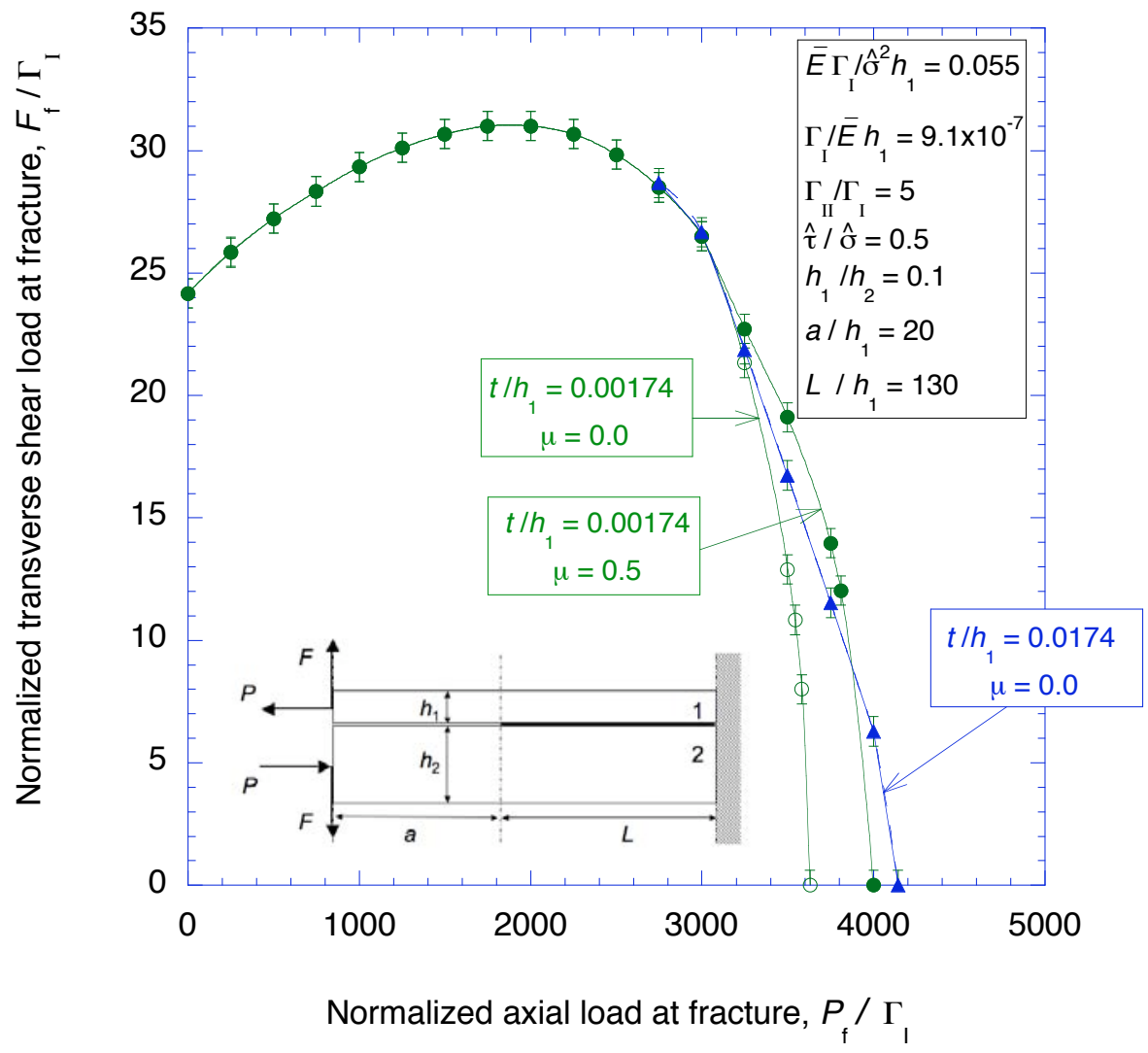

Figure 11: (a) 


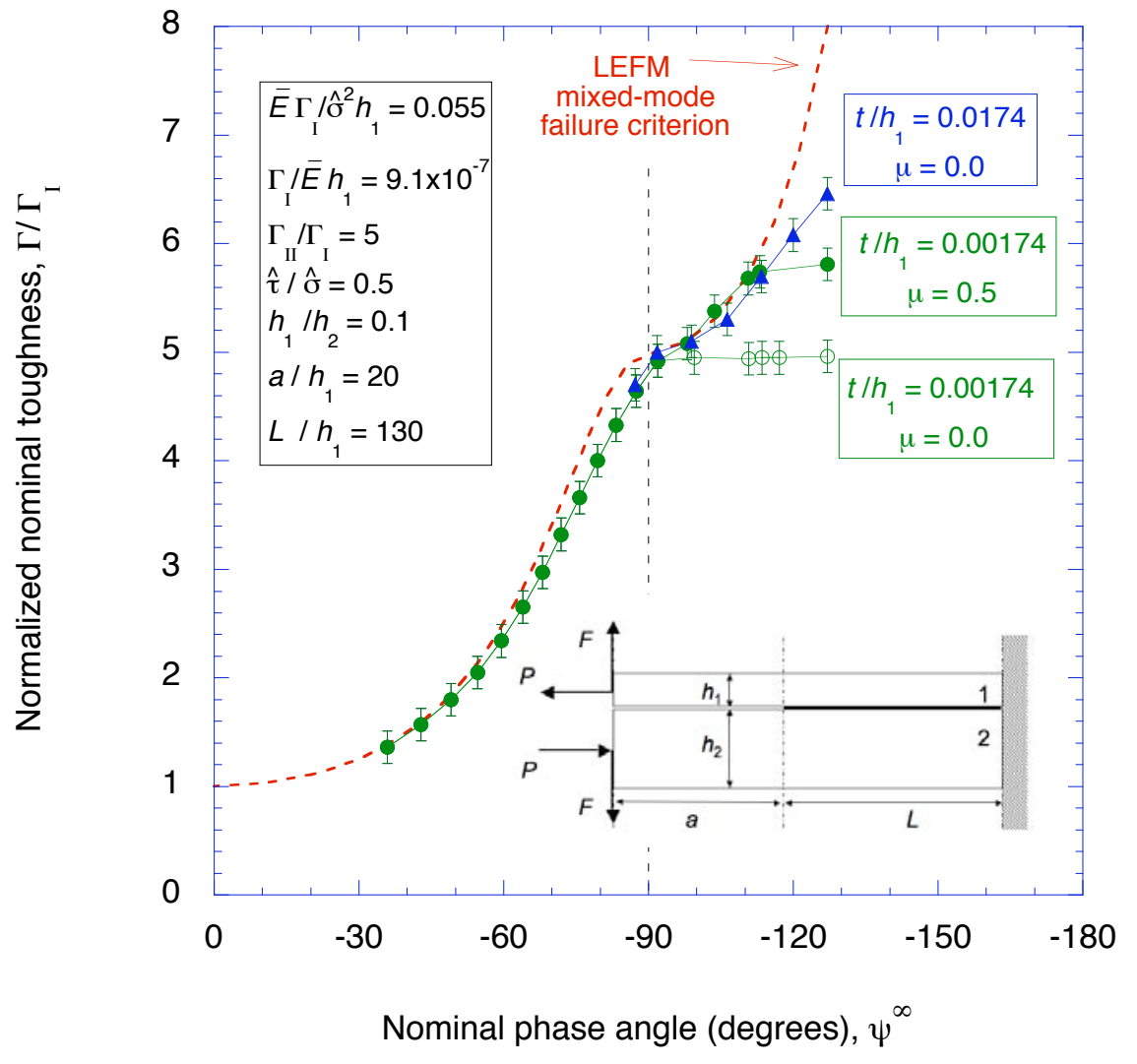

Figure 11: (b) 


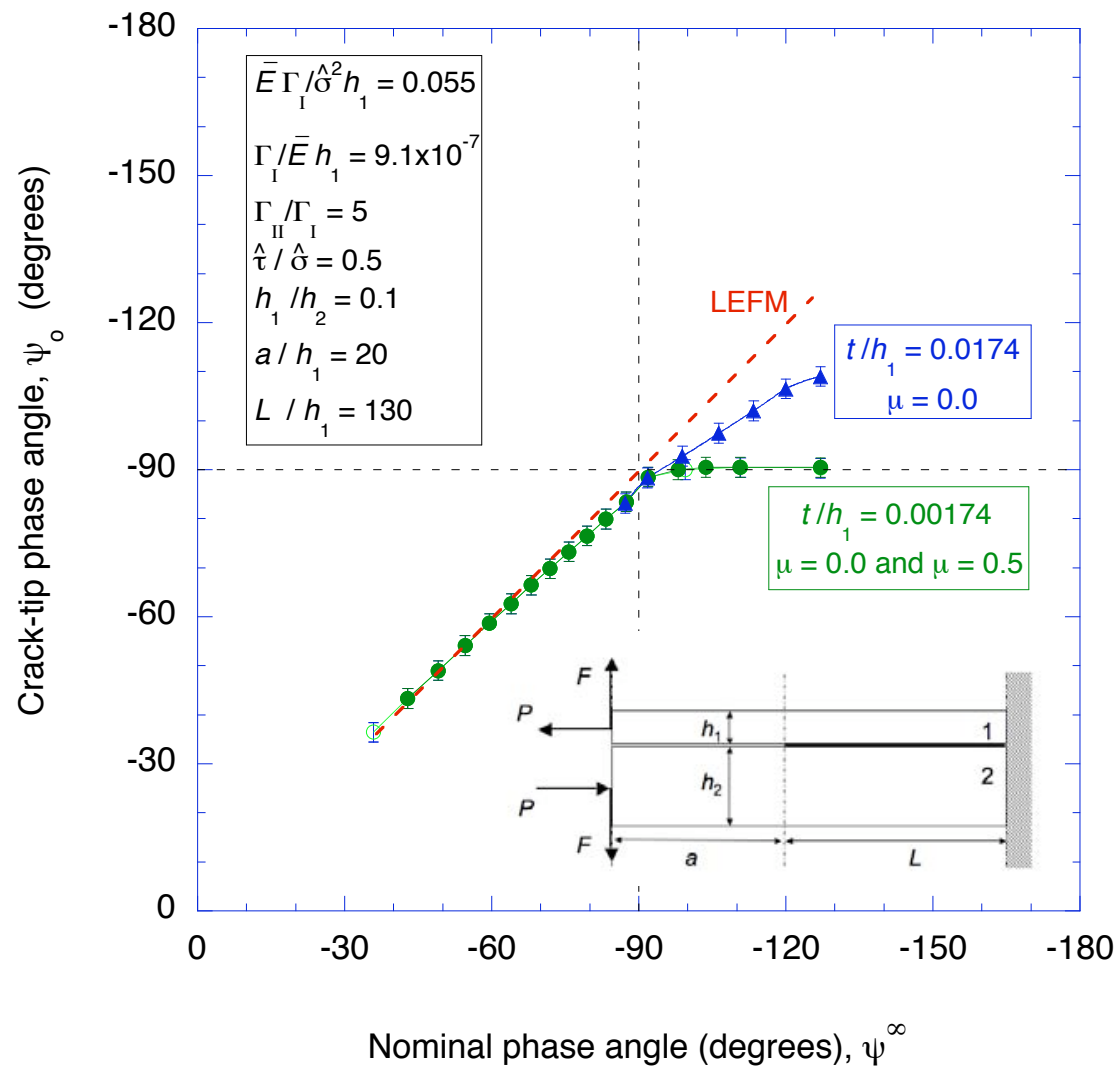

Figure 11: (c) 


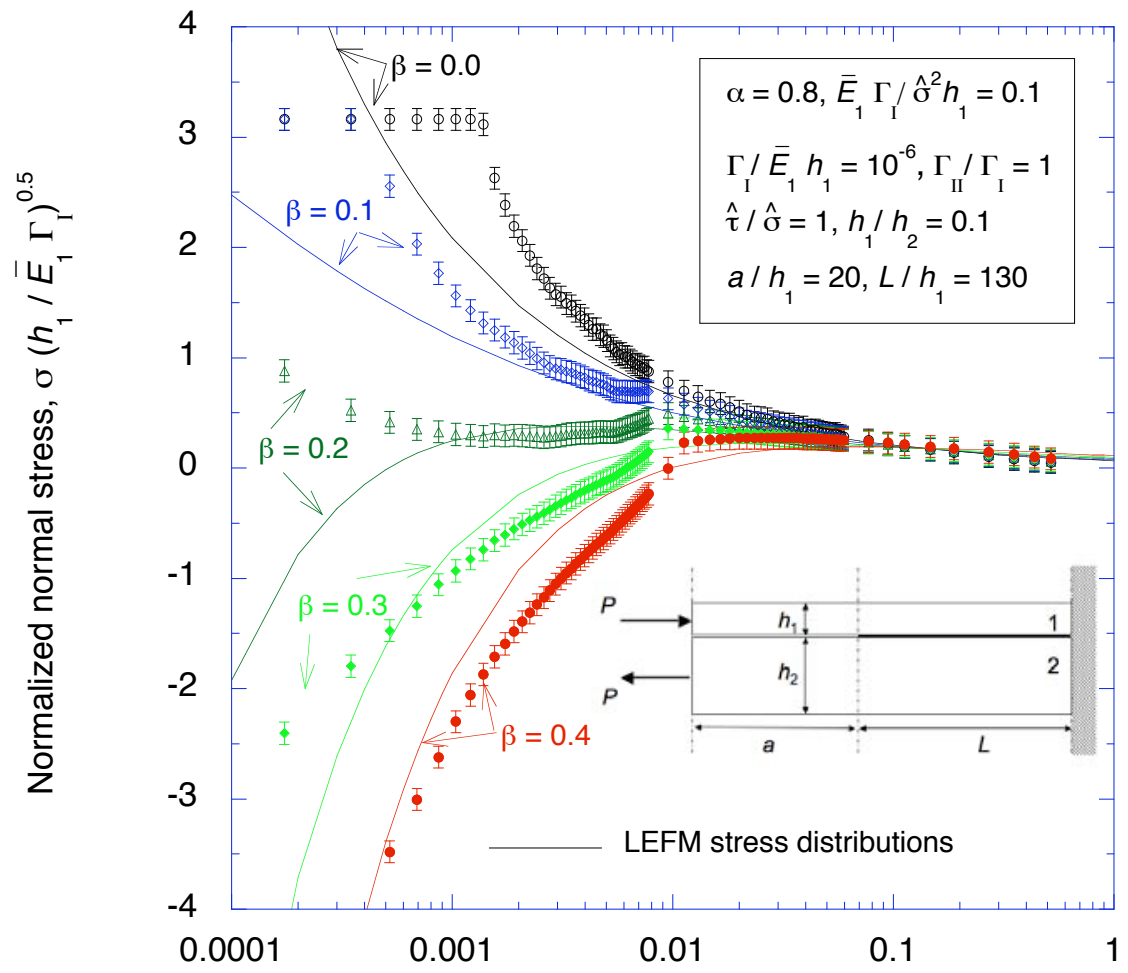

Normalized distance ahead of crack tip, $r / h_{1}$

Figure 12: (a) 


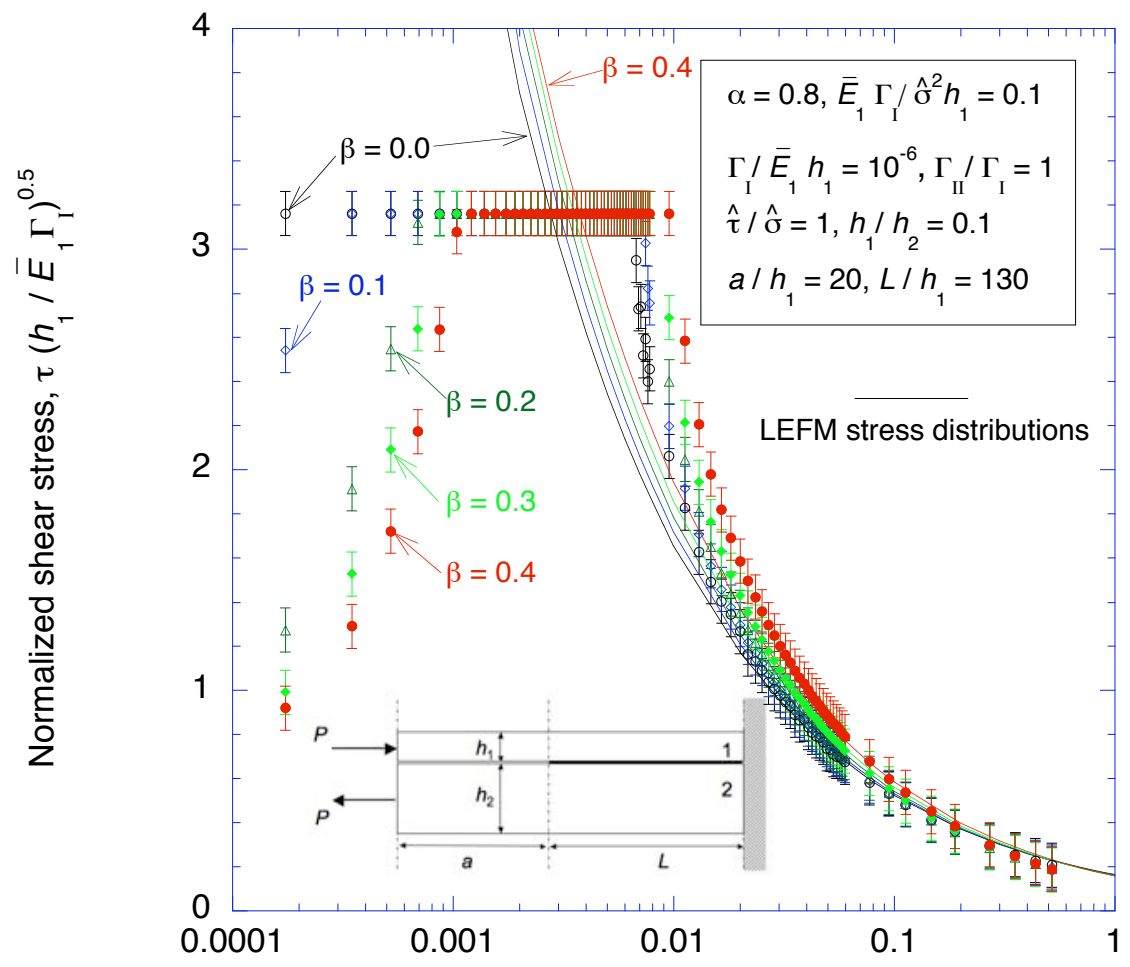

Normalized distance ahead of crack tip, $r / h_{1}$

Figure 12: (b) 


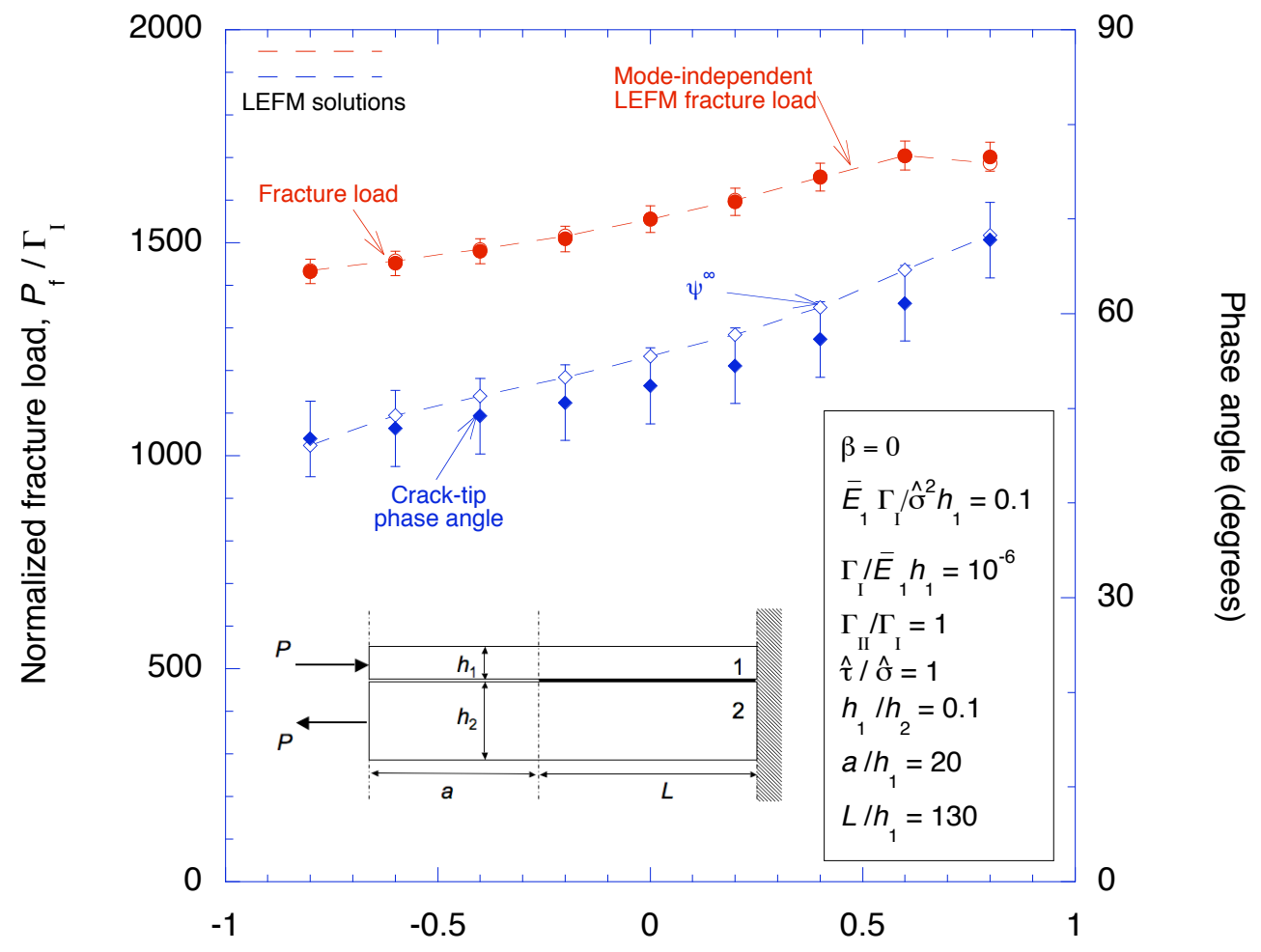

Elastic mismatch, $\alpha$

Figure 13: (a) 


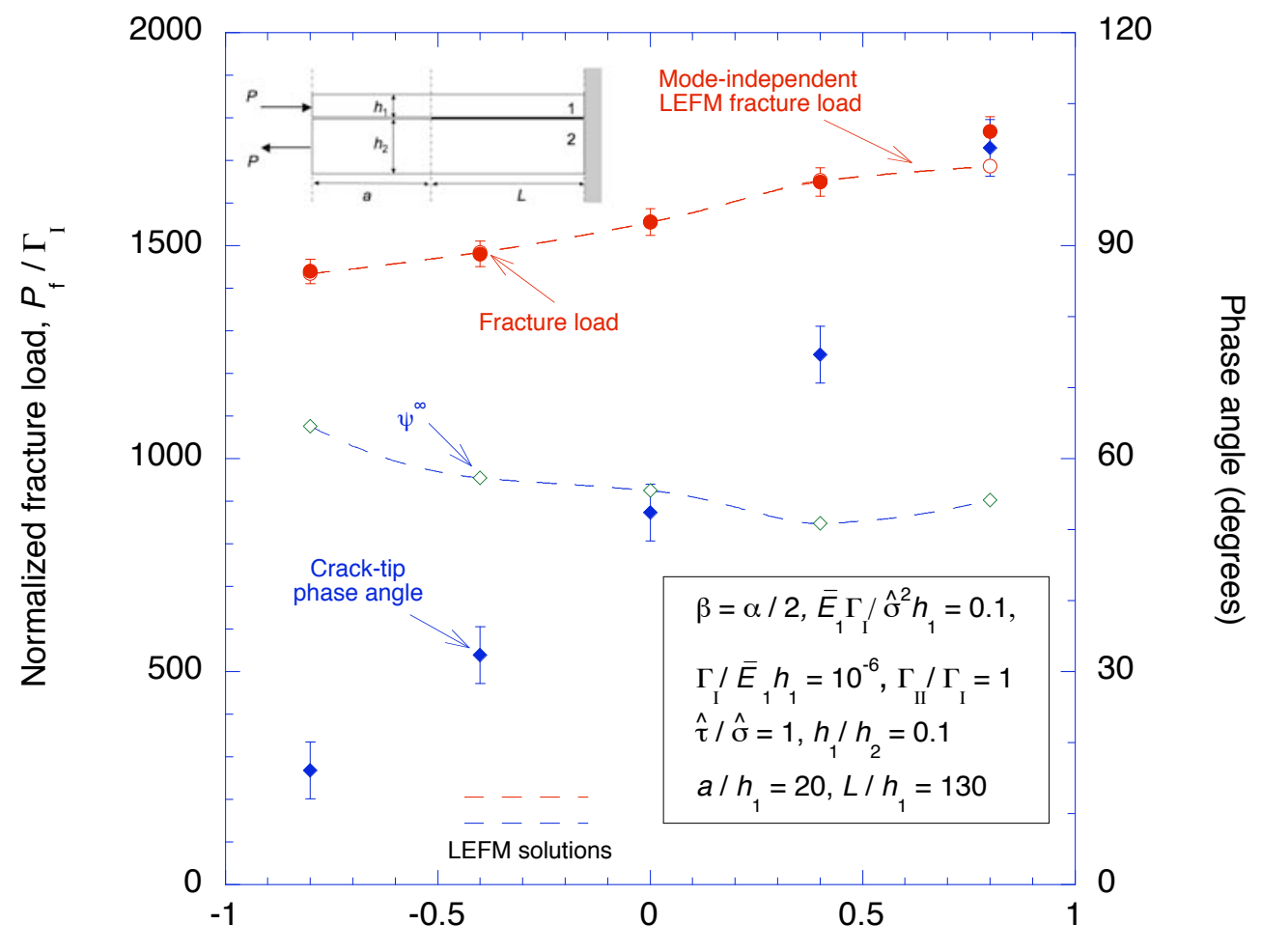

Elastic mismatch, $\alpha$

Figure 13: (b) 


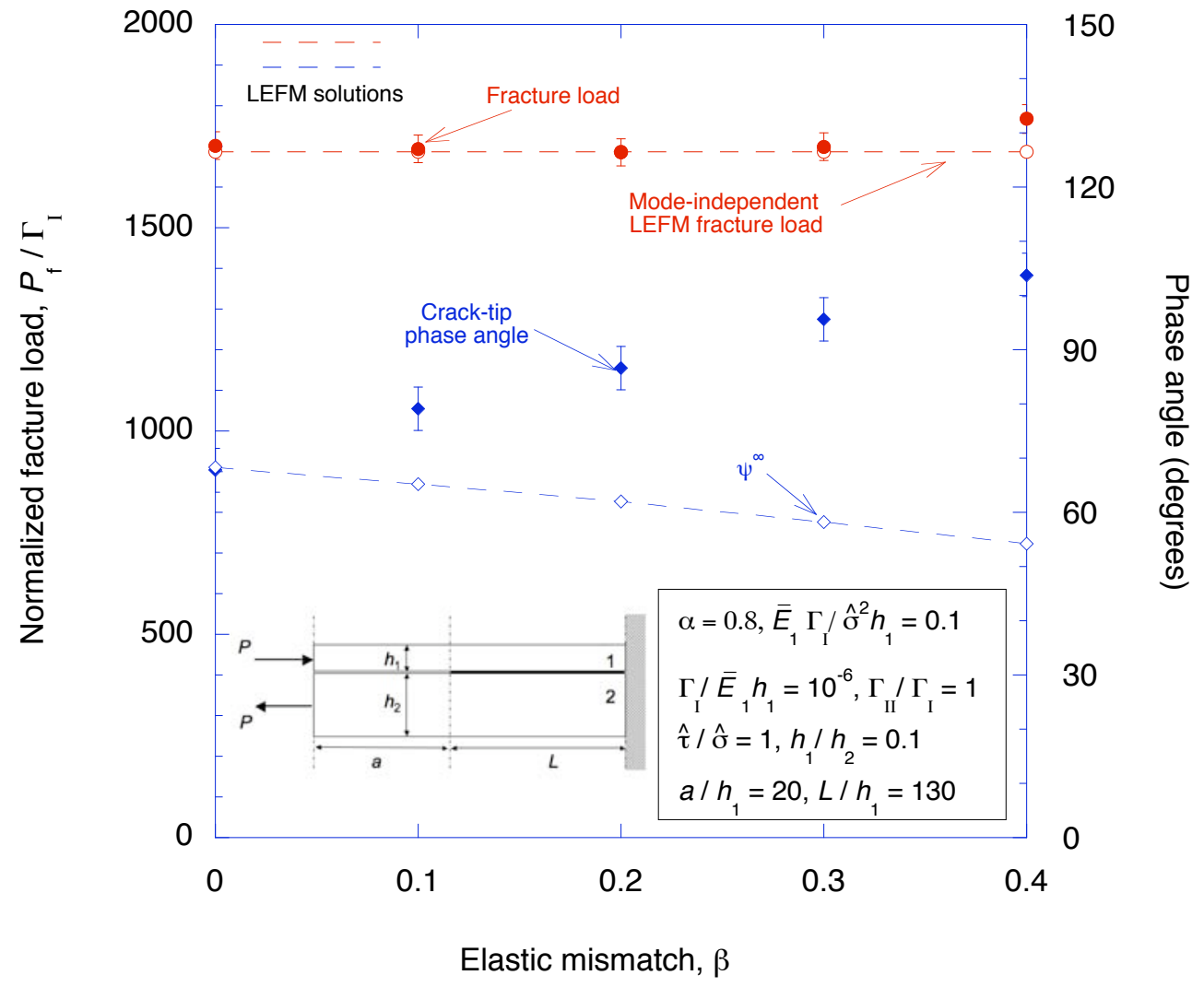

Figure 13: (c) 


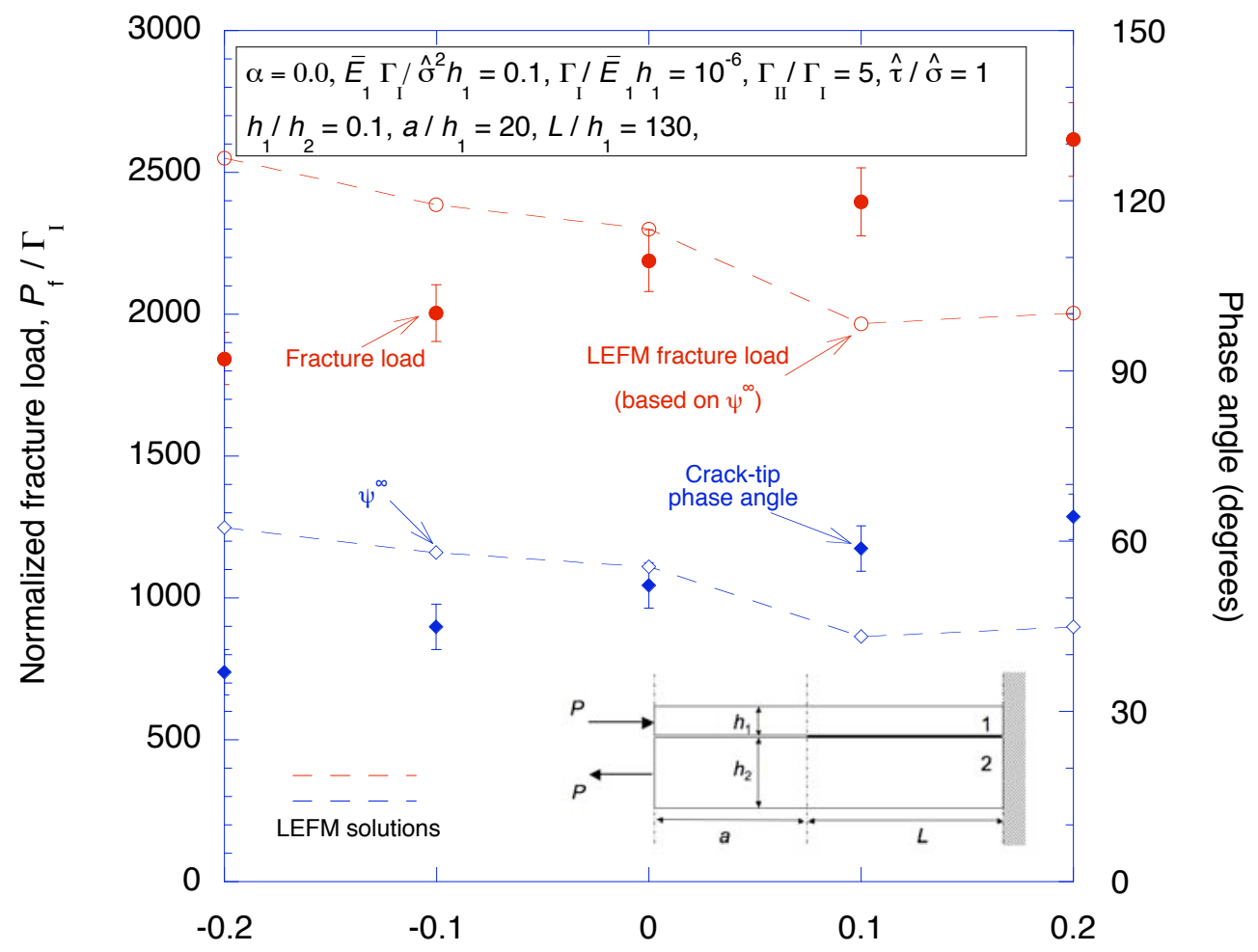

Elastic mismatch, $\beta$

Figure 14: 


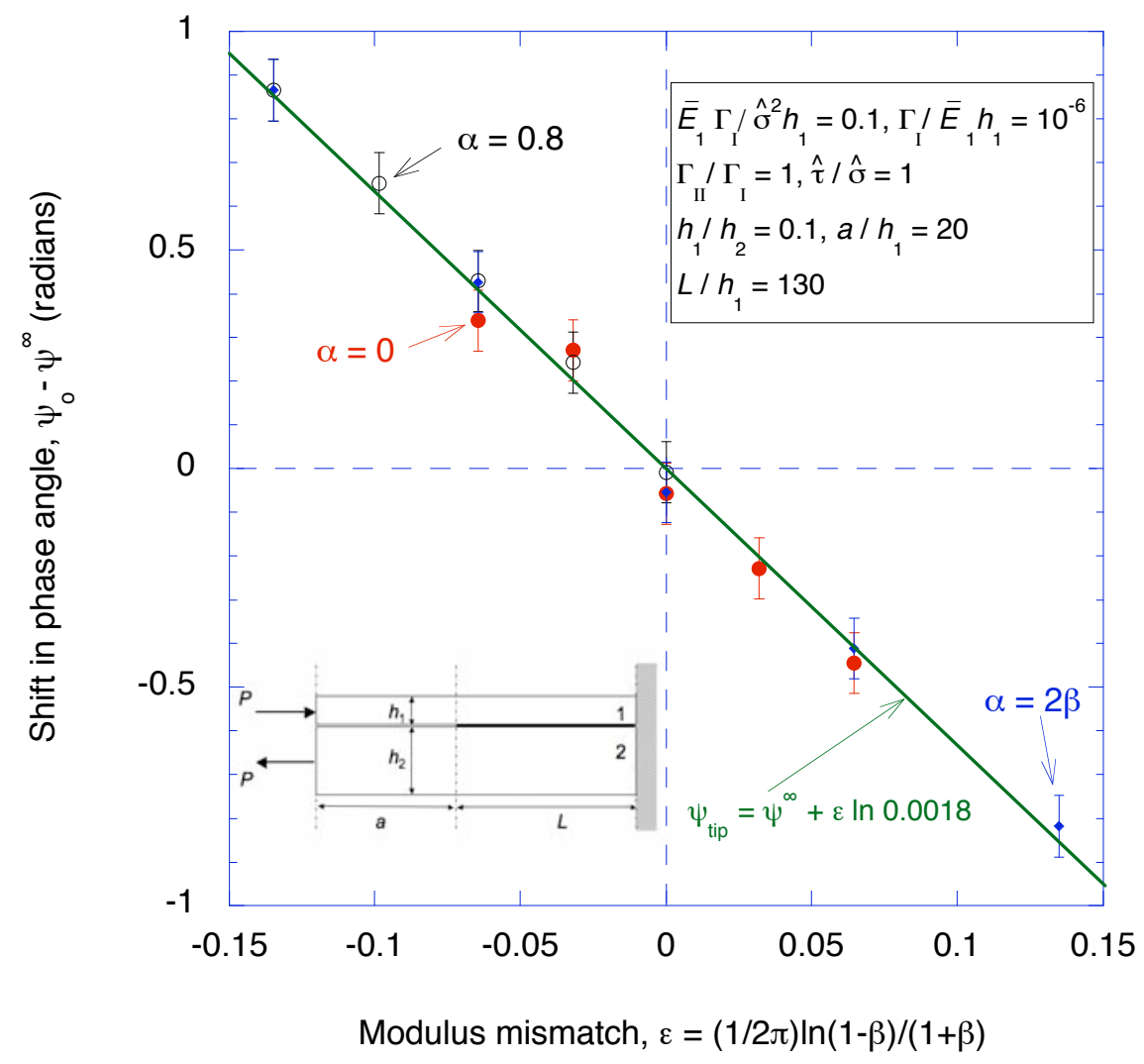

Figure 15: 


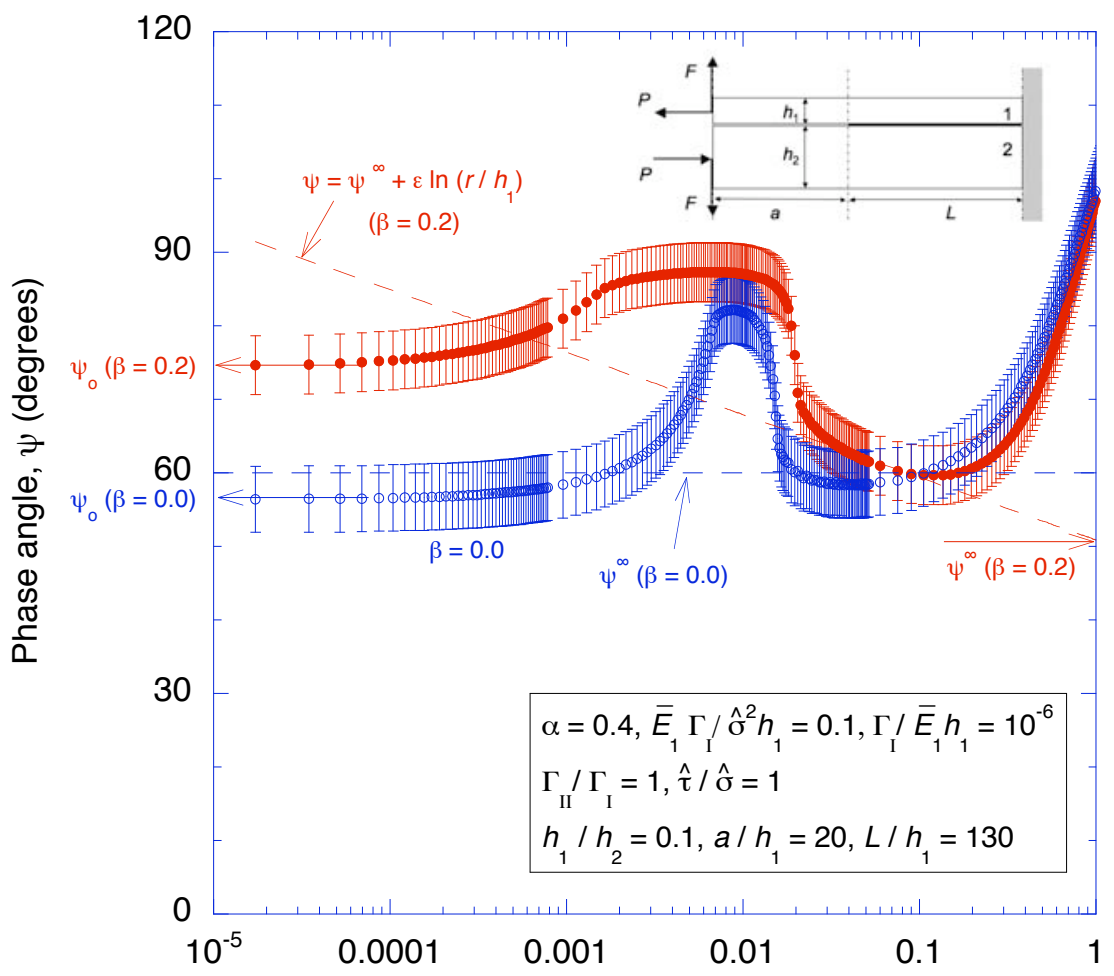

Normalized distance ahead of crack tip, $r / h_{1}$

Figure 16: 